\title{
Current Status of the Hypothesis of a Claustro-Insular Homolog in Sauropsids
}

\author{
Luis Puelles \\ Department of Human Anatomy, University of Murcia, Institute of Biomedical Research (IMIB-Arrixaca), El Palmar, \\ Spain
}

\begin{abstract}
Keywords
Homology · Hodology · Bauplan · Pallium models . Genoarchitecture · Claustrum · Insula - Sauropsids . Mammals
\end{abstract}

\begin{abstract}
The author previously worked extensively on the broad problem of the evolution of the vertebrate pallium. He proposed various Bauplan models covering at least gnathostomes, based in the definition of a set of pallial sectors with topologically invariant positional relationships and distinct molecular profiles. Out of one of these models, presented as the "updated tetrapartite pallium model," a modified definition of the earlier lateral pallium sector (LPall) concept emerged, characterizing it in mammals as an unitary claustro-insular transitional (mesocortical) complex intercalated between the neocortex or dorsal pallium (DPall) above and olfactory cortex or ventral pallium (VPall) underneath. A distinctive molecular marker of the early-born deep claustral component of the LPall was found to be the transcription factor Nr4a2, which is not expressed significantly in the overlying insular cortex or in adjoining cortical territories. Given that earlier comparative studies had identified molecularly and topologically comparable VPall, LPall, and DPall sectors in the avian pallium, an avian $\mathrm{Nr} 4 \mathrm{a} 2$ probe was applied, aiming to identify the reportedly absent avian claustro-insular complex. An early-born superficial subpopulation of the avi-
\end{abstract}

an LPall that expresses this marker selectively through development was indeed found. This was proposed to be a claustrum homolog, whereas the remaining Nr4a2-negative avian LPall cells were assumed to represent a possible insular homolog. This last notion was subsequently supported by comparable selective expression of the mouse insular marker Cyp26b, also found restricted to the avian LPall. Some published data suggested that similar molecular properties and structure apply at the reptilian LPall. This analysis was reviewed in Puelles et al. [The pallium in reptiles and birds in the light of the updated tetrapartite pallium model. 2017]. Four years on, the present commentary discusses some international publications accrued in the interval that touch on the claustro-insular homology hypothesis. Some of them are opposed to the hypothesis whereas others corroborate or support it. This raises a number of secondary issues of general interest.

(c) 2021 S. Karger AG, Basel

\section{Introduction}

Historically, comparative anatomists generally conceived the telencephalic claustrum as a structure exclusive of mammals, although some authors such as Källén [1951, 1953, 1955, 1962] and Kuhlenbeck [1924, 1973] conceived abstract pallial models which implicitly allowed the possibility of non-mammalian claustrum homologs (Fig. 1a, b). Striedter [1997] and Bruce [2007, 


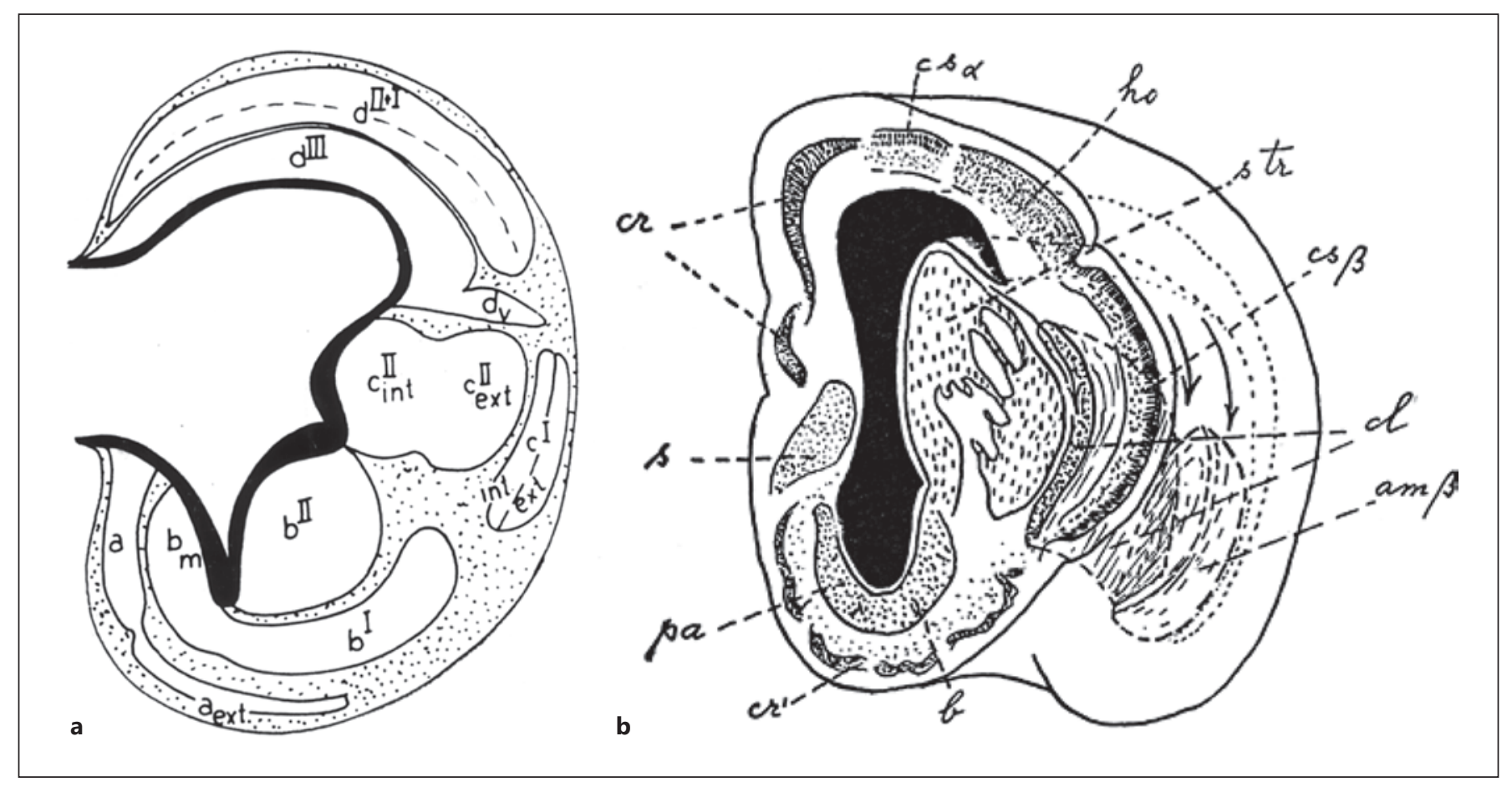

Fig. 1. a Detail of Figure 8 from Källén [1955] illustrating an early schema of amniote pallium and subpallium subdivisions. The claustrum, represented here by $\mathrm{d}_{\mathrm{v}}$, is mapped on the pallial side, without an insular cover. Note the bilayered olfactory cortex $\left(c^{\mathrm{I}}\right)$ was placed superficially to the striatum ( $c^{\mathrm{II}}$ ext and $c^{\mathrm{II}}$ int). If we imagine the olfactory primordium to have slipped out through the interstice separating the claustrum from the striatum, we have a close enough image of the present day VPall and the unitary LPall claustro-insular complex. The rest of the cortex was included in dI, dII, dIII. The pallidum forms the $b^{\mathrm{I}}, \mathrm{b}^{\mathrm{II}}$, and $\mathrm{b}_{\mathrm{m}}$ complex. The area a continuous with $\mathrm{a}_{\text {ext }}$ marks the septum and the diagonal band. b Tri-dimensional drawing from Kuhlenbeck [1924] illustrating pallial and subpallial components in a generalized "lower" mammal, and his idea of a joint origin of the striatum and the claustrum within his D1 pallial region, as well as the caudal continuity of the claustrum with the pallial amygdala $(\operatorname{am} \beta$; nucleus amigdalae beta). Note there is an arrow next to the ventricular zone marking where claustral neurons come from, which is the small domain medial to the lateral ventricular angle which we now conceive to be pallial (compare ventral pallium of Fig. 2), whereas the underlying striatal area is clearly subpallial. Interestingly, the claustrum is depicted as lying deep to the olfactory cortex ( $\operatorname{cs} \beta$; cortex striatus beta), that is, it actually refers rather to the dorsal endopiriform nucleus; the insula is not identified, but probably would lie ventrally within the neocortex (ho; cortex homogeneticus of Brodmann). This shows Kuhlenbeck clearly supported at least at this time a claustral origin independent from the insula.

solateral (lateropallial) and ventromedial (ventropallial) components, within novel LPall and VPall pallial sectors distinguished on the basis of presence versus absence of Emx1 expression. With the dorsolateral claustro-endopiriform component we actually were referring to what we now identify separately as the claustrum proper (found within LPall) plus the dorsal endopiriform nucleus, also known classically as "ventral claustrum" (the latter actually lies in the adult in the VPall after it migrates tangentially from the claustral primordium at the LPall; see Fig. 2) [Puelles, 2014; Watson and Puelles, 2017]. With the ventromedial claustro-endopiriform component we were referring to what we now identify as the claustral subplate (which is actually a true LPall component; see 
Fig. 2) [Puelles, 2014] plus the ventral endopiriform nucleus (which is in contrast truly ventropallial; see Fig. 2). The misunderstanding was due to wrong assumptions about the radial glial structure of this pallial region, a point that was cleared in Puelles [2014].

Another occult problem that hampered at this time our thinking in dealing with possible homologies of the avian LPall was that we failed to contemplate existing data indicating that the avian pallium stratifies in an outsidein pattern, just the opposite of the mammalian inside-out pattern. This means that avian claustrum-homolog cells, having extremely precocious birthdates, like the mammalian counterparts, had to be initially at the brain surface in both mammals and birds, remaining permanently so in birds but only transiently in mammals, where the developing insular cortex gradually covers the preexisting claustrum (Fig. 3, 4a-c) [Puelles, 2014; Puelles et al., 2016a].

As it turned out, another significant problem in our early definition of the mammalian LPall [Puelles et al., 1999,2000 ] was that we thought it included a lateral part of the olfactory cortex jointly with the deep "dorsolateral claustro-endopiriform complex." This wrong conclusion came from the belief that the main claustrum and the EPd were traversed radially by a packet of radial glia ending pially at the lateral part of the olfactory cortex (the medial part corresponding instead to VPall). This radial organization is actually what classic authors looking at adult brains had described (see literature review and radial glia evidence in Puelles [2014]). However, this assumption turned out to be false, since glia processes passing through the claustrum actually end distinctly at the insular pial surface (Fig. 5) [Puelles, 2014].

Finally, we followed Holmgren [1925] and other classic authors in the assumption of a claustroamygdaloid complex, in the sense that the amygdalar basolateral anterior nucleus was thought to derive from LPall, like the claustrum [Puelles et al., 2000; Medina et al., 2004). This assumption has also turned out to be false [Puelles, 2014; Puelles et al., 2019a, b; Garcia-Calero et al., 2020, 2021; Garcia-Calero and Puelles, 2021].

I suspected for a longtime that a distinct entity such as the mammalian claustrum had to have evolutionary antecedents in non-mammals, though they might be cryptic in histologic appearance, as I thought was the case of monotremes that reportedly lack a distinguishable claustrum (see the literature review and comments in Puelles [2014]). Serendipitously, an extensive embryonic collection of Nurr1 (Nr4a2) in situ preparations cut in three section planes was prepared in my lab for other purposes



Fig. 2. Schema extracted from Puelles [2014] and Watson and Puelles [2017] that displays the adjacent VPall and LPall as studied in the mouse. The course of radial migration occurring within the VPall (green colored) essentially produces the olfactory cortex (or system, with rostral and caudal differentiations), jointly with two deep nuclear remnants of the ventropallial migration stream (VMS), the ventral endopiriform nucleus (VEn), and the bed nucleus of the external capsule (BEC). Radial migration occurring in the adjacent LPall (orange color) puts in place first the principal claustrum population (ClP), which is transiently subpial, and then the covering insular cortex (Ins). Cells with molecular properties similar to those of claustral cells migrate tangentially during development: some enter the subplate deep to the claustrum (these are the earliest born neurons, even earlier than claustral ones) and proceed dorsalward deep to the dorsopallial incipient cortical plate (subplate; black arrow). It is a myth that subplate cells occupy this position before the cortical plate starts to form [see Puelles, 2014]; others, called Arimatsu cells according to their discoverer, migrate subpially into the neighboring parietal neocortex, where the primordium is invaded when it is formed only by prospective layer 5 and 6 neurons (superficial black arrow). Later, the younger neocortex layers develop over them, so that in the adult, Arimatsu cells typically occupy parietal deep layers 5/6a; finally, a third claustral population migrates ventralward into VPall, forming the dorsal endopiriform nucleus (DEn) deep to the dorsal part of piriform layer 3 (white arrows).

(work by Antonia Alonso) and I spent time examining it. I easily corroborated the conclusion of Arimatsu et al. [1994] that this gene is an excellent marker of adult mammalian claustrum and dorsal endopiriform nucleus neurons. I should say that it also labels other quite distinct nearby or distant telencephalic pallial and subpallial sites, in part due to apparent tangential migration of claustral populations [Puelles, 2014]. This material, amplified sub- 
Fig. 3. Claustral cells expressing the marker $\mathrm{Nr} 4 \mathrm{a} 2$ are precociously postmitotic, which is why they are found at the brain surface at the earliest stages of development (no other cells are born concurrently at the specific LPall sector where they are born). Here coronal sections from E13.5 mouse and E7 (HH32) chick are compared for very similar presence of subpial Nr4a2-positive early claustral cells, illustrating their similar topological position relative to VPall (N; nidopallium), LPall (M; mesopallium), and DPall $(\mathrm{H}$, hyperpallium), and how both primordia lie at the brain surface. The asterisk in a marks Nr4a2-positive subplate cells migrating out of the claustral primordium into the DPall cortical subplate. Note the ventral migration of dorsal entopeduncular neurons has not started yet. The black arrowhead in $\mathbf{b}$ marks a few $\mathrm{Nr} 4 a 2$-positive claustral cells starting to migrate into the hyperpallium (DPall). c More advanced E10 (HH36) chick embryo telencephalic cross-section showing the superficial Nr4a2-positive claustrum population restricted to the LPall or mesopallium. Other mesopallial structural details are identified. $\mathrm{CL}$, claustrum; HA, apical hyperpallium; $\mathrm{HD}$, densocellular hyperpallium; M, mesopallium; MI, intermediate mesopallial nucleus; N, nidopallium; SCL, subclaustral stratum; SPall, subpallium.

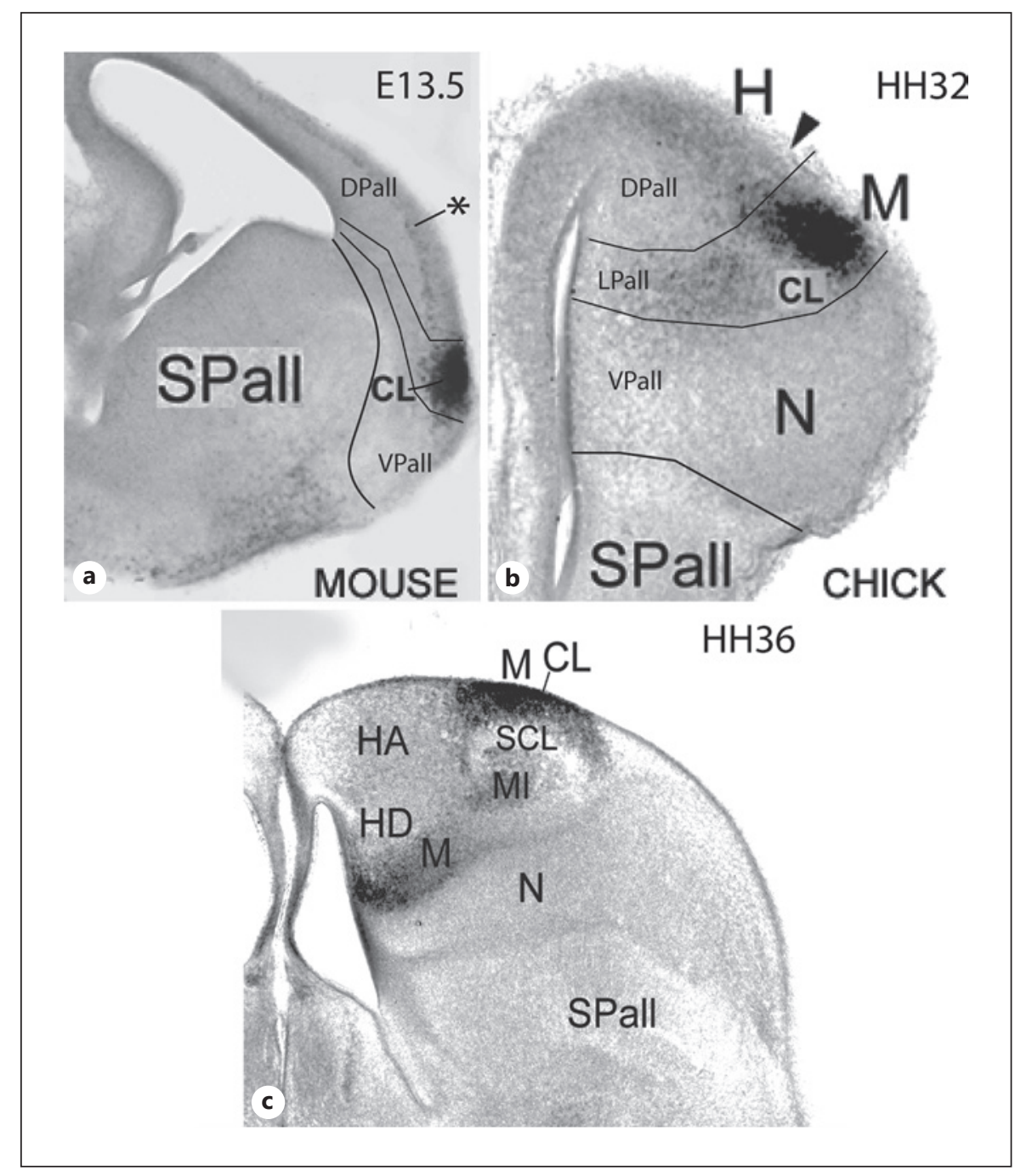

sequently by analysis of radial glial processes testing how they pass through the Nr4a2-positive claustrum and at which pial surface area they end, led to my new LPall concept in the updated tetrapartite pallium model. This model produced solutions for all the mentioned problems at the LPall which had handicapped our initial tetrapartite pallial model (Fig. 2, 3a, 4a, b). The updated version returns to classical notions of claustro-insular joint development in mammals and to the conclusion that the claustrum is accordingly topologically and developmentally tied to the insula/perirhinal mesocortex, and not to either the olfactory allocortex or the pallial amygdala, although this last notion only became completely clear more recently [Puelles, 2014; Puelles et al., 2019a, b; Garcia-Calero et al., 2020, 2021; Garcia-Calero and Puelles, 2021]. This progress illuminated the various errors in the Puelles et al. [2000] pallium model and generated a sounder mod- el update changing appropriately the VPall, LPall, and DPall notions (Fig. 2; comments on the previous errors are also found in Puelles [2014, 2017], Puelles et al. [2016a, 2017], and Watson and Puelles [2017]).

The VPall was redefined as an Emx1-negative and partially Dbx1-derived pallial sector that contains the whole olfactory cortex $(\mathrm{OB}, \mathrm{AOA}$, Pir; note a dorsal part of this cortex lacks Dbx1-derived cells) [Puelles et al., 2016b], plus the underlying ventral endopiriform nucleus (EPv) and the newly distinguished bed nucleus of the external capsule (BEC; Fig. 2, 4a-f) [Puelles, 2014; Puelles et al., 2016b]. Contrarily, the LPall, which now could be identified at very early stages by its selective claustral $\mathrm{Nr} 4 \mathrm{a} 2$ expression, a precocious and spatially circumscript differentiation (Fig. 3a), was redefined in Puelles [2014, 2017] as a claustro-insular complex that in the adult contains deeply the precociously developed claustrum, cov- 


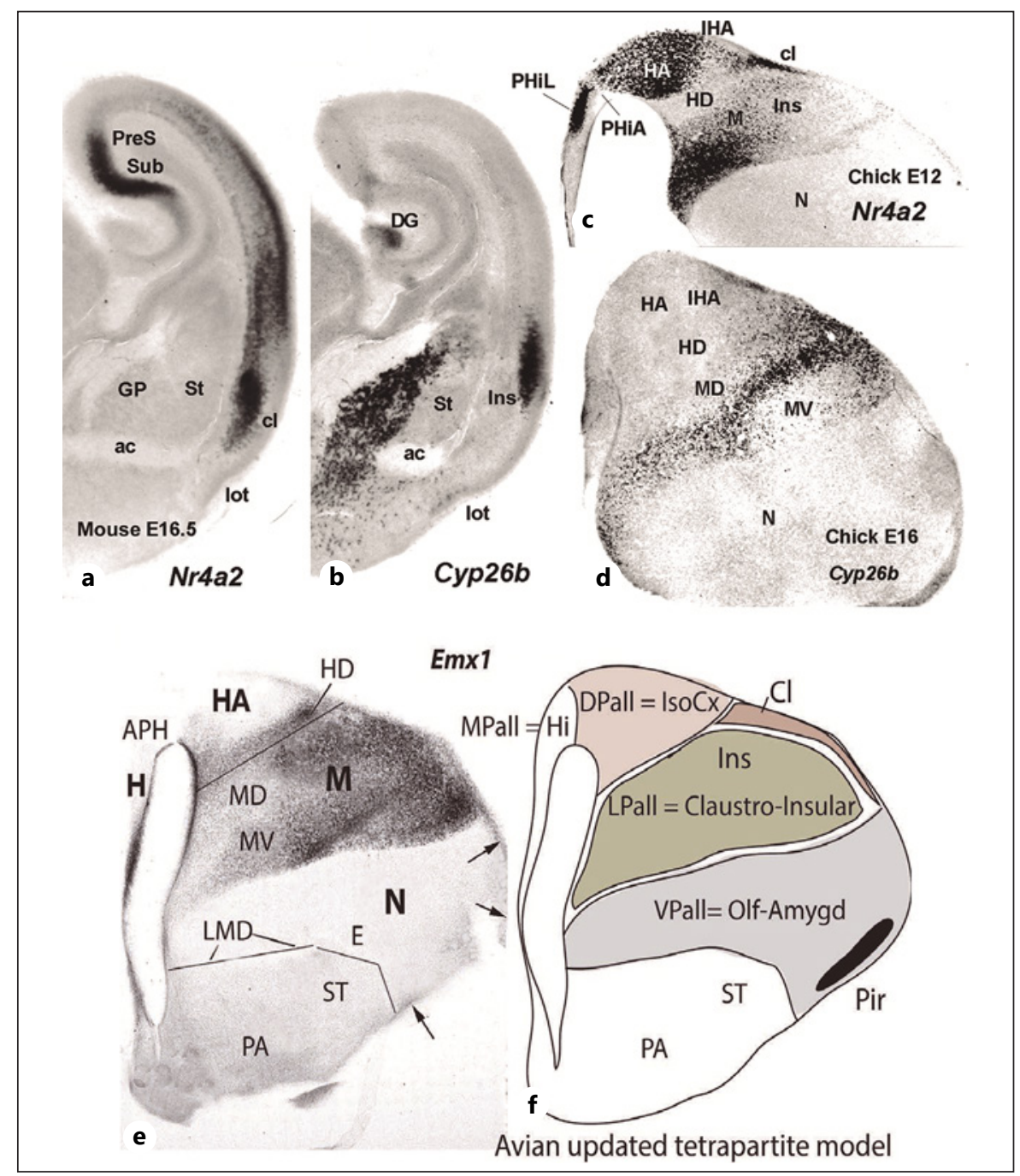

Fig. 4. Intermediate stages (E16.5 mouse in $\mathbf{a}, \mathbf{b}$ and chick stages E12 and E16 in c, d) serve to compare Nr4a2 as a claustral marker (a, c) and Cyp26b as a partial insular marker (b, d) in both species. Note, however, that the section planes for mouse and chick are not comparable. We see in a that the mouse claustrum (cl) has a deeper position, being partially covered by the insula (Ins) in $\mathbf{b}$. The insular marker also labels the pallidum in the subpallium, medially to the striatum (GP, St in $\mathbf{a}, \mathbf{b}$; there is a corresponding pallidal expression in the chick; not shown), and both markers label distinct hippocampal cell patches (PreS, Sub, DG in $\mathbf{a}, \mathbf{b}$; PHiL in c). In contrast, in the chick the claustral cells in (c) lie subpially (cl) and the insular cells (Ins) aggregate underneath; there is some $\mathrm{Nr} 4 a 2$ signal periventricularly but the Ins cells are largely $\mathrm{Nr} 4 a 2$-negative. The insular marker shown in $\mathbf{d}$ spreads across the radial extent of the insular domain but is irregularly distributed (it does not label the whole insula in the mouse either). The primary labeled locus in chicken for both markers is the mesopallium or LPall (M), but many migrated claustral cells have selectively invaded the HA by this stage, eschewing the interposed HD (HA, HD in c). The insular cells do not seem to migrate into other parts of the pallium and remain strictly within $\mathrm{M}$. e, f Images illustrating the molecular boundary between mesopallium and nidopallium. The microphotograph in e illustrates meso- pallial Emx1 expression in an E12.5 chick embryo (cross-section at midlevel of the hemisphere. HD, HA, parts of DPall; APH, H, parts of MPall; ST, PA, parts of subpallium; MD, MV, parts of LPall; N, E, parts of VPall; LMD, lamina medullaris dorsalis, i.e., the palliosubpallial boundary). The schema in $\mathbf{f}$ reproduces the same section interpreted according to the updated tetrapartite pallium model, featuring VPall (gray background) and LPall (green and pink) as molecularly distinct parts separated by a differential Emx2 signal within the old LP (compare with e). The DPall appears in a light pink background, limited medially by the white MPall. The LPall shows the postulated division into superficial claustrum (CL; pink background) and inner insula (Ins; green background). Note alternative names for all sectors. The black subpial domain of VPall represents the piriform cortex. ac, anterior commissure; APH, perihippocampal area; cl, claustrum; DG, dentate gyrus; E, entopallium; GP, globus pallidus; $\mathrm{H}$, hippocampus; HA, apical hyperpallium; $\mathrm{HD}$; densocellular hyperpallium; IHA, intercalated lamina of apical hyperpallium; Ins, insula; lot, lateral olfactory tract; M, mesopallium; MD; dorsal mesopallium; MV, ventral mesopallium; $\mathrm{N}$, nidopallium; PA; pallidum; PHiA, apical perihippocampal area; PHiL, lateral perihippocampal area; PreS, presubiculum; Pir, piriform cortex; St/ST, striatum; Sub, subiculum. 
ered by the subsequently developed, largely Nr4a2-negative insular/perirhinal cortex (InsCx; Fig. 4). The latter reveals in the mouse an inside-out layering pattern similar to that found in the neighboring neocortex. The insular/perirhinal cortex selectively displays in some layers $C y p 26 b$ transcripts among other LPall-selective markers [Puelles, 2017] (Fig. 4a, b; e.g., see Lhx2 in the Allen Developing Mouse Brain Atlas at E15.5; this selectivity is lost at older stages). The DPall notion was modified by excluding the mesocortical insula/perirhinal cortical areas from its domain, an aspect already contemplated in previous literature [e.g., Zilles and Wree, 1985]. Whereas the main claustrum appears under the conventional insula, a caudally diminishing claustral extension which lies deep to the perirhinal cortex and is hardly distinguishable cytoarchitectonically is observed with the Nr4a2 marker. Both insular and perirhinal claustral primordia give rise to ventrally migrated Nr4a2-positive dorsal endopiriform populations [Puelles, 2017; Watson and Puelles, 2017; Puelles et al., 2019a]. The caudal EPd component appears in the adult just lateral to the lateral amygdalar nucleus, and is also referred to as the caudal EP (EPc). The boundary between InsCx and PRhCx (and between rostral and caudal EPd formations) lies roughly at the anterior commissure level [Puelles et al., 2019a, b]. Interestingly, InsCx and $\mathrm{PRhCx}$ are functionally interconnected [Ramos, 2020].

The claustral/insular/perirhinal complex thus substitutes for the old "claustro-amygdaloid complex" previously conceived in the literature, which was negated by my Nr4a2 observations [Puelles, 2014]. There is no Nr4a2 expression in the mouse amygdala at any developmental

Fig. 5. a, b Selective identification of the claustral primordium using $\mathrm{Nr} 4 \mathrm{a} 2$ in situ hybridization on floating sections at E16.5 in the mouse with secondary RC2 immunoreaction of the radial glial cells in the same sections. This aimed to test the hypothesis that the claustrum relates to the insula against the hypothesis that it relates to the olfactory cortex. In the first case, radial glial fibers are expected to pass from left to right across the claustrum into the insula (from the depth into the insular pia); in the second case they should pass from the top downwards towards the olfactory cortex at the bottom. These sections were oriented in a plane oblique by some 45 degrees relative to the brain midline, found empirically to encompass the glial fibers in the insular-claustrum area most completely. Both high-magnification examples $(\mathbf{a}, \mathbf{b})$ show the immature claustrum and overlying insula to the right. It can be clearly seen that the radial glial fibers approach the claustrum from the depth (to the left and above) and course through it into the insula. No fiber in any of the sections examined in several brains was seen to cross the claustrum ventrally towards the olfactory cortex. Similar data were obtained in E14.5 and E18.5 specimens as well. stage or in the adult, though the field conventionally identified as chick amygdala contains a sizeable patch of $\mathrm{Nr} 4 a 2$ expression that is always separate from the avian LPall expression domain. In the mouse, the caudal claustrum-derived caudal EPd is the sole claustral family component that approaches the pallial amygdala at the level of the lateral nucleus, but it does not penetrate the pallial amygdala complex proper [Garcia-Calero et al., 2020].

The selective precocious identification of the earlyborn mouse claustrum population with the $\mathrm{Nr} 4 \mathrm{a} 2$ marker (Fig. 3a) suggested it might be worthwhile examining

the correlative chicken LPall sector defined by Puelles et al. [2000] (which had been renamed "mesopallium" in the meantime; Reiner et al. [2004]) for a possible Nr4a2-positive claustrum homolog. We expected to detect a precociously born LPall cell population expressing Nr4a2 selectively. Note that in birds early born pallial cells occupy superficial positions due to the outside-in stratification pattern of the avian pallial mantle [Tsai et al., 1981; Striedter and Keefer, 2000].

The predicted avian "claustrum" population did exist precisely where we expected, namely occupying the superficial stratum of the mesopallium (avian LPall; Fig. 3b, c), which lies intercalated topologically between the olfactory VPall (nidopallium), ventrally, and the DPall or hyperpallium, dorsally (Fig. 3b, c) [Puelles et al., 2016a, 2017; Puelles, 2017]. Previously published evidence from the Molnar lab suggested that a corresponding early lateropallial Nr4a2-positive population (with identical relative position) exists likewise in turtle embryos [Wang et al., 2011]. This was corroborated subsequently by Tosches et al. [2018] (see below). The avian mesopallial cell population thought to be field-homologous with the set of mammalian claustral populations (both non-migrated and tangentially migrated cells) even includes at advanced embryonic stages tangentially migrated derivatives comparable to the mouse counterparts [Puelles et al., 2016a; Puelles, 2017]. A Nr4a2-positive population migrates tangentially from the LPall into the apical hyperpallium part of the DPall (see the asterisk and arrowhead marked elements in Fig. 3a, b; i.e., these are thought to be possible

Fig. 6. a Image illustrating a corroborating experimental test of the postulated claustral tangential migrations in the chick. This is a cross-section at the middle level of the telencephalic hemisphere of an E11 chick embryo, showing in red fluorescent immunoreactive expression of the general pallial marker Tbr 1 and in blue background cellular staining with DAPI. The green-labeled cells correspond to neurons derived from progenitors electroporated selectively at the LPall anlage at E4 (shortly before the birth of claustral cells) with a fate-mapping transgenic construct developed by García-Moreno et al. [2014]. White lines indicate the boundaries between the four pallial sectors within the updated tetrapartite pallium model (MPall, DPall, LPall, and VPall). The maximal number of construct-labeled cells occurs superficially within the LPall or mesopallium (labeled as claustrum; CL), while later born deeper mesopallial strata (Ins) remain unlabeled. A stream of labeled cells distinctly penetrates subpially the DPall, corresponding to the claustral cells interpreted as colonizing the apical hyperpallium, possibly homologous with mammalian subplate cells and/or Arimatsu deep layer cells (compare schemata in b). A different subpial stream penetrates the VPall and numerous cells aggregate just under the piriform cortex (Pir), building an EPd homolog struc- homologs of mouse Nr4a2-positive subplate cells that invade the DPall). Another subpial Nr4a2-positive cell group enters tangentially the VPall, reaching a position deep to the avian ventropallial olfactory cortex (i.e., apparently an EPd homolog; see Fig. 6). These results satisfy the three classic criteria of homology: invariant topologic position within a Bauplan (LPall within the tetrapartite pallium model), same developmental origin (presumed shared pallium patterning mechanism into VPall, LPall, and DPall), and presence of special characteristics (precocious birthdates, singular genoarchitecture ( $\mathrm{Nr} 4 a 2$ signal), and comparable tangentially migrated derivatives; Jacobshagen [1925, 1927]; Kuhlenbeck [1927, 1973]; Remane [1952]; Puelles and Medina [2002]; Medina [2007]; Nieuwenhuys and Puelles [2016]).

Our avian claustrum findings were presented at the First Symposium on Evolution of the Cerebral Cortex (Toledo, Spain, 2015) and were published in The Journal of Comparative Neurology the following year [Puelles et al., 2016a]. Our homology hypothesis was also presented at the VIII European Conference on Comparative Neurobiology (Munich, 2016), jointly with our preliminary data mapping the Cyp26b insular marker (Fig. 4b, d) in the (deep) avian mesopallium population produced after the claustral one [Puelles, 2017]. We reviewed afterwards various aspects of the possible reptilian claustro-insular homolog complex [Puelles et al., 2017], and presented also some experimental evidence supporting the described tangential migratory movements (Fig. 6) [Watson and Puelles, 2017].

ture (compare schemata in b). Note that piriform cortical populations (Pir) at the VPall surface are hardly labeled. The distribution of all of these construct-labeled cells coincides with independently mapped Nr4a2 cells [Puelles et al., 2016a]. b Schematic comparison of adult pallial composition in terms of tetrapartite model analysis across adult reptiles, birds, and mammals (dashed pallial sector boundaries), including postulated claustro-insular components at the LPall or migrated out of it (modified from Puelles et al. [2016a]). The MPall is color-coded red and the DPall is green; the LPall is doubly coded, with claustral Nr4a2-positive elements in violet and insular elements in light blue; related tangential migrations into the DPall and the VPall are illustrated as prolongations of the main violet claustral domain; the VPall is color-coded yellow, with an orange-colored piriform cortex cell plate. The ventricular cavity is in black. There is remarkable conservation between sauropsids and mammals of the general positional pattern of claustrum-derived elements, with the exception that the claustrum lies subpially in sauropsids and deep to the insula in mammals (see text). BEC, bed nucleus of the external capsule; DVR, dorsal ventricular ridge; EPd, dorsal endopiriform nucleus (or homolog); EPv, ventral endopiriform nucleus; SP, subplate.

(For figure see next page.) 
We knew that our postulate of a sauropsidian claustroinsular complex at the mesopallium was unexpected in the field and realized that colleagues would not react unanimously with applause (although in science unexpected results are supposed to be of highest interest). We assumed that colleagues holding positions on avian pallium opposed to our updated tetrapartite pallial model and its claustro-insular LPall component in the mouse [Puelles, 2014] would speak up, pointing out objections to our hypothesis, if only because a mesopallial avian and reptilian claustrum - with a possible insula homolog attached - significantly seem to falsify their conjectures, pointing future comparative pallium studies in a completely different direction (I refer here to the followers of
Karten's notion of a cryptic neocortical nature of component populations of the sauropsidian dorsal ventricular ridge; Karten [1969, 2015]; e.g., Reiner et al. [2005]; Butler et al. [2011]; Suzuki and Hirata [2013, 2014]; Briscoe and Ragsdale [2018]; review in Puelles et al. [2013, 2017, 2019b], and below). On the other hand, we hoped that colleagues basically satisfied with the theoretic and evidence-based background of our tetrapartite molecular pallium model might express at least their interest in the novel hypothesis. This group is possibly divided into: (1) the less-informed ones still working with the outdated Puelles et al. [2000] pallium model version, possibly including Wullimann [2017a, b]; (2) those newly adapted to the objectively more realistic Puelles [2014] tetrapartite




model update, and (3) those preferring a similar divided pallium with more than four sectors, for example Medina and collaborators (see below).

I suspect that the pallial experts of both signs, i.e., those either supporting or opposing in principle our tetrapartite pallium model, were initially equally surprised by the Puelles et al. [2016a] report, because a claustro-insular joint developmental concept was new in comparative analysis of cortex evolution, even though this idea is not devoid of conceptual antecedents in pioneering classic cortical studies (review in Puelles [2014]). This concept also involves the Cinderella-like insular mesocortex (i.e., a transitional cortex domain intercalated between allocortex and isocortex, sensu; Dart [1934] and Sanides [1969]; review in Puelles et al. [2019a]). This distinct domain of mammalian pallium is rarely mentioned (or considered) in current evolutionary pallium models (but see Striedter [1997]). Various modern authors apparently assume that "cortex" just means "neocortex."

I confess that the subject of a developmental claustroinsular complex was surprising also for me, for different reasons. I had always assumed the correctness of existing embryologic literature which pictured the mammalian claustrum as developing once the insula is in place, by migrating directly into its deep adult position (e.g., Bayer and Altman [1991]; Medina et al. [2004]; see review in Puelles [2014]). Before writing the Puelles [2014] book chapter I needed weeks of daily exposure to the Nr4a2 data and substantial reflection before I realized it meant that the earliest cells expressing $\mathrm{Nr} 4 \mathrm{a} 2$ lie at the brain surface, apparently as a part of the early cortical plate. The meaning, of course, was that claustral cells are born before the ulteriorly suprajacent insular cortical cells, and thus primarily occupy the cortical surface before migrating insular neurons subsequently displace them passively inwards as they themselves access the same locus of the cortical plate. Rather than a separate migration pathway for claustral cells [Bayer and Altman, 1991], there apparently existed just a standard radial pathway for the claustro-insular complex. I checked the corresponding published cell birthday data in rodents (amply commented in Puelles [2014]: see also the recent analysis by Fang et al. [2021] in the rat) and verified that claustrum neurons (as well as subplate cells and the dorsal endopiriform nucleus) were long known to be born before insular ones (that is, before any cortical plate pyramidal cells). This heterochrony of finally inside-out stratified cell populations of the claustro-insular complex suggested a relationship of both claustrum and insula cells with a particular packet of radial glial processes passing successively through the claustrum and the insula (and not through the claustrum and any part of the olfactory cortex, contrary to what $\mathrm{Pu}$ elles et al. [2000] assumed). I was able to verify this prediction, using specific labeling of the claustral primordium with the Nr4a2 marker and radial glia immunostaining (Fig. 5) [Puelles, 2014].

I can thus understand the reserve on the side of those not considering yet a sauropsidian claustrum or a claustro-insular homolog in their pallium evolution schema. Time is needed to examine how the claustrum homology hypothesis affects your assumptions, beliefs, and previously published conclusions. Published reactions have been slow in coming (and there was practically no private feedback, a bad omen) but reactions finally started to appear 2-3 years later. The present occasion seems appropriate for reviewing the main responses, including comments on Atoji et al. [2018], Briscoe and Ragsdale [2018], Desfilis et al. [2018], Tosches et al. [2018], and Biegler et al. [2021], with passing mention of some other correlative papers.

\section{Comments}

\section{J.M. Wild}

Atoji and Wild [2012] previously studied the connections of the avian mesopallium, the pallial domain we now regard as the avian claustro-insular LPall complex [Puelles, 2017]. However, they did not target specifically the superficial locus occupied therein by the claustrum homolog (Atoji and Wild [2012]; comments in Puelles et al. [2016a, 2017]). Wild eventually contacted me by email to comment that he had revised the possible connections of the postulated mesopallial avian superficial claustrum homolog with the Wulst (DPall homolog candidate), and he had not found them. At the time he had not yet reported these observations, but he apparently referred to experiments subsequently published in Atoji et al. [2018] on the projections and afferences of the intermediate and denso-cellular parts of the Wulst (HI, HD), which also included control injections in the apical hyperpallium (HA) and the frontal lateral nidopallium (NFL). Unfortunately, given that the emphasis of the paper was on $\mathrm{HI}$ / HD connections, the photographic evidence offered in this report does not focus sufficiently on the mesopallium. The authors comment specifically on the claustral homology hypothesis and conclude that their results do not support the existence of theoretically expected claustral bidirectional connections with the DPall (taken as a neocortex field homolog). It might be argued that some 
cells retrogradely labeled at the HA after CTB retrograde tracer was placed at HI or HD may represent claustral cells "displaced" by the tangential LPall-into-DPall migration we described into the Wulst [Puelles et al., 2016a]. I suppose that I also have the same license accorded to Karten [1969] and followers, who can postulate that homologous cell populations moved into other regions (in this case labeled with a rather selective gene probe). On the other hand, BDA injections in the $\mathrm{HI}$ did generate abundant anterograde-labeled fibers that coursed through (and probably also connect with) the superficial and deeper mesopallium before reaching more distant targets (Atoji et al. [2018]; their Fig. 6a-c). Control injections placed at the NFL may have labeled jointly the closely neighboring superficial mesopallium (e.g., their Fig. 7d), or the laterally migrated "endopiriform" claustral neurons (Puelles et al. [2016a]; photograph in Puelles [2017] reproduced here as Fig. 6a). Similarly, retrogradely labeled cells identified in the "HD" after a CTB injection at the HA (Atoji et al. [2018]; their Fig. 9d) may include some elements in the closely adjacent claustrum. This is all rather inconclusive. Accordingly, I think that the specific experiments we suggested in Puelles et al. [2017] that should be centered on the claustrum are still needed to throw proper light on this issue. There will appear below some other comments on this topic.

With regard to the final allusive remarks in the discussion of Atoji et al. [2018], I must explain that I do not regard conservation of connections as a necessary criterium for homology, irrespective of whether this occurs or not in the case of the avian claustrum (functional evidence cited below suggests that such connections probably exist). It is indeed satisfactory and quite interesting to see conserved connections between homologous brain areas of distant species, a not rare circumstance, but I think that lack of similar connections in separate lineages is strictly no proof against the respective postulated homologies of the source and target territories (pallial domains in this case). For instance, the avian main sensory trigeminal nucleus uniquely connects directly via the quintofrontal tract with a rostral nidopallial target (n. basorostralis), but this hodological singularity of birds does not alter in any way the homology of the avian main sensory trigeminal nucleus with its counterparts in other vertebrate species in which these systematically connect instead with a thalamic target. The main criteria of topological position in the Bauplan and comparable mode of development (both related to alar rhombomere 2 in the hindbrain and the ganglion and sensory root of the trigeminal nerve) support sufficiently this homology, whereas the ascending hodological discrepancy is taken as irrelevant by everybody, as far as I know.

My rationale for considering connections a secondary and non-essential comparative criterium is that every neuronal connection depends on a complex series of navigational and synaptogenetic developmental events affecting both the growing axons en route (namely their adhesive, permissive vs. blocking, or repellent contacts) and the relevant final postsynaptic sites. These obligatory developmental events involve specific serial molecular interactions with a variety of cells, depending on the relative position of the growth cone along its route, which can easily vary in evolution. This is one reason why I never understood the hypothesis of Karten [1969] that given cell populations may change their topologic positions via embryonic tangential migrations and nevertheless reproduce thereafter precisely their ancestral connections, now starting from a different place; neither Karten himself nor any of his followers has ever demonstrated or explained such hodological conservatism. The complex combinatorial character of axonal navigational interactions along their course may easily suffer changes in evolution due to minor protein mutations accompanying evolutionary change, leading to qualitatively varied connections without elimination of the original regional homologies of the source and target cell populations, as plausibly occurs in the mentioned case of the avian trigeminal main sensory nucleus. I can thus contemplate with interest, if needed, an avian claustrum homolog that has lost its cortical connections and has found a different way to be biologically useful. However, this may not be needed, since other novel data I will comment upon below relative to the reptilian claustrum serendipitously imply that the latter has whatever cortical connections are essential for its main function, strictly comparable to that recently described in the mouse. The same function seems equally required in birds, so that I believe that the essential claustro-cortical connections must somehow be there.

I think that Owen's [1848] apt definition of homology still holds: "The same organ in different animals under every variety of form and function." This is sometimes criticized as being tautological, but note it evades mentioning a requirement of "similarity" (a concept wrongly touted in more recent comparative work); it just demands "sameness." I believe that Owen correctly understood comparative "sameness" to refer to corresponding components of a Bauplan or archetype in any shape or functional adaptation, even though he could not know that such morphostatic features of organic forms are maintained by the genome, heredity, and development across evolution. 
In the brain, variety of form and function usually appears in the connections, not in the much more conserved molecular regionalization of progenitor areas (i.e., patterning of pallial sectors in this case, but see also any other part of the brain). The significantly corroborated (although possibly still imperfect) tetrapartite pallium model (please check the step forward contemplated in Puelles et al. [2019a]) holds that vertebrates largely share a common molecular patterning of their telencephalic pallium. It is in this aspect (i.e., the fundamental local genomeenforced Bauplan) where "cerebral sameness" resides (frequently involving field-homology phenomena: Puelles and Medina [2002], Medina [2007], Nieuwenhuys and Puelles [2016], irrespective of some prestigious contrary opinions, e.g., Striedter and Northcutt [2020]). Epiphenomenal connections or functions are in this regard, in my opinion, essentially variable evolutionary characters.

This rationale based on strongly conserved patterning of the brain and potentially evolutionarily variable hodology diverges from the message of Faunes et al. [2015], who seem to reject every genomic, evolutionary, or developmental morphostatic possibility, and solely approve $o b$ server-defined hodological similarity. I regard this thesis as an attack upon all we know about evo-devo regulatory mechanisms and brain anatomy/neuroembryology (see other comments below in the section dedicated to the work of Tosches and colleagues).

\section{Desfilis and L. Medina}

In a study of the lizard pallium, Desfilis et al. [2018] recently formally endorsed the sauropsidian claustrum homology hypothesis, even though they presented it within an alternative hexapartite model of the sauropsidian pallium that introduces some differential conceptual and terminological peculiarities (Fig. 7a, b). Desfilis et al. [2018] distinguish in the lizard telencephalon nominally the standard MPall, DPall, LPall, and VPall pallial sectors of my updated tetrapartite model [Puelles, 2014], tagging them MP, DP, LP, and VP, to which the authors add novel dorsolateral and ventrocaudal pallial sectors (DLP, VCP; Fig. 7a, b). In order to introduce the DLP component - which includes their claustrum homolog - they change significantly in their schema the definitions of the lizard DP and LP territories when compared to my current model (note their VCP is not problematic, and will not be discussed here, because it is restricted to the pallial amygdala, which I regard as a separate pallial field; Puelles et al. [2019a, b]).

The conceptual changes introduced in this work essentially are as follows: (1) they reduce the DP (by elimi- nating our caudal hyperpallium or HC, and transferring the equivalents of avian HI/HD hyperpallial areas to the intermediate DLP; iDLP; Fig. 7a); (2) they locate their LP within my VPall, and ascribe to it a lateral local part of the olfactory cortex as well as the ventral part of the standard atlas mesopallium (MV); (3) they rename the dorsal part of my claustro-insular LPall as their new intermediate DLP domain (iDLP). I do not agree with any of these operations (further discussed below) because they do not seem sufficiently supported by existing data on birds and mammals. A major problem of this study is that neither the Nr4a2 marker nor any other selective claustral marker was employed to define the lizard claustrum homolog; this means that their identification of the claustrum is highly tentative. Moreover, the lizard $\operatorname{Lh} x 9$ data that are adduced instead cannot be obtained in chick or mouse embryos (as they acknowledge themselves). In any case, the latter are not sufficient justification for separating the unitary claustro-insular complex into moieties not recognized in other tetrapods: avian MD (within iDLP) and MV (within LP and connected to olfactory cortex).

One might think that Desfilis et al. [2018] agree with Wullimann's [2017a, b] contention that the LP term should be saved on historic reasons for a lateral part of the olfactory cortex (see my answer in Puelles [2017]). They also seem to have approximated his specific penta-partite model proposal by putting the ventral part of my claustro-insular unit (avian MV) within the "olfactory pallium" sphere (their "LP" lying within my VPall), while pushing the upper part of my claustro-insular unit (avian $\mathrm{MD}$ ) into the new DLP sector. Confusingly, Wullimann [2017a, b] apparently independently suggested the same "DLP" (dorsolateral pallium) name, which he applied to a cortical domain found either inside or next to the neocortex or dorsal pallium (not so the lizard iDLP of Desfilis et al. [2018]). The resulting postulate of 5 cortical pallial sectors in the lizard (if we exclude the exclusively amygdalar VCP domain) is nevertheless surprisingly very similar to Wullimann's solution (see comments in Puelles [2017]).

\section{The DPall Issue}

Desfilis et al. [2018] do not accept our recent definition of a novel caudal part of the avian hyperpallium (our HC within an expanded Wulst or DPall; Fig. 12 in Puelles et al. [2016a, 2017]). They rather consider our HC domain as a caudal part of their DLP (cDLP), held to be homologous to the mammalian entorhinal cortex. The avian and reptilian $\mathrm{HC}$ was postulated by us (on topologic grounds and some molecular observations) as possibly homolo- 


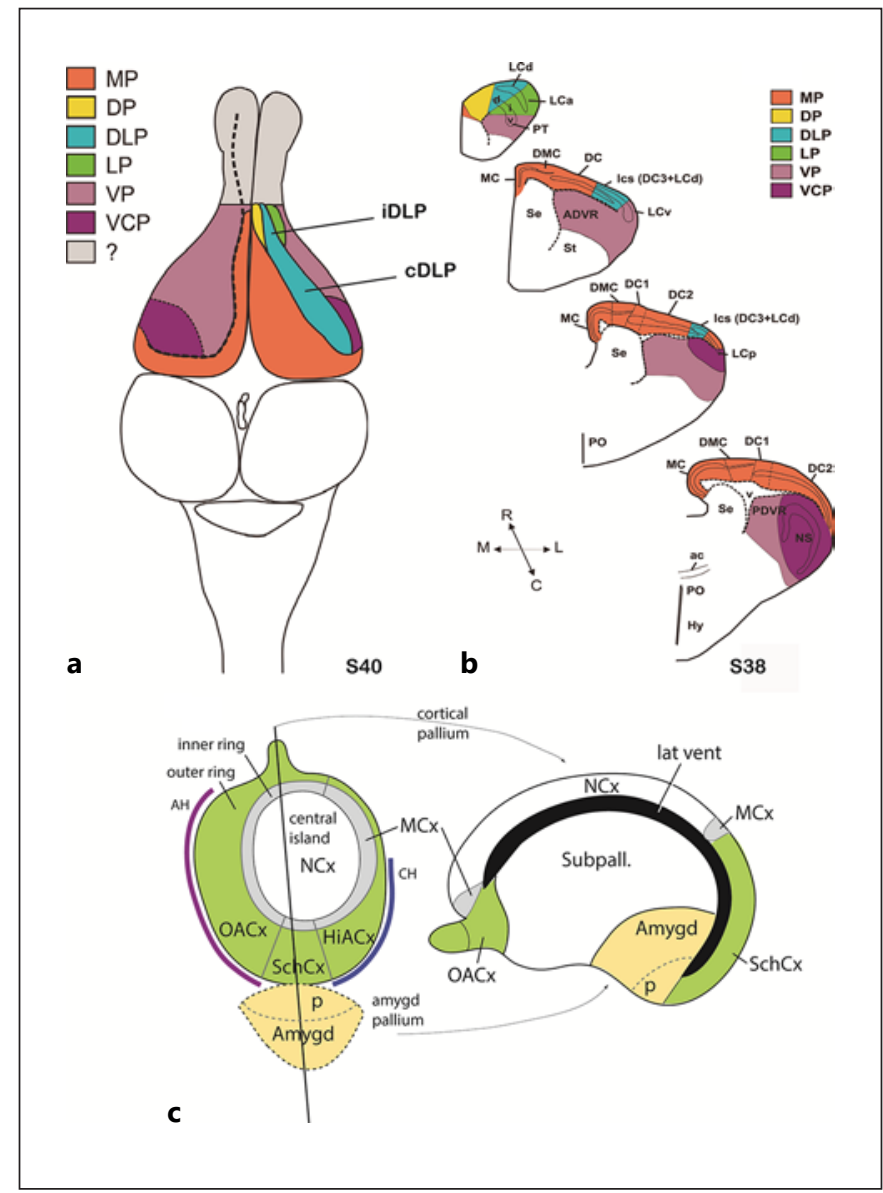

gous to the mammalian temporal neocortex. This HC domain introduced for the first time a sauropsidian DVRindependent homolog of the topologically caudalmost mammalian neocortex, which was missing from the usual concept of the avian Wulst, widely held to represent a neocortex homolog. Karten's followers (e.g., Reiner et al. [2005]) characteristically described temporal cortex topographically in mammals as the "lateral neocortex" (although it is manifestly topologically caudal, according to telencephalic morphogenesis). These authors speculatively held that this part of the neocortex originates in mammals from the "mammalian DVR" (i.e., largely my VPall) via undemonstrated mass tangential migrations (VPall into DPall) or, alternatively, from a conjectured VPall progenitor field duplication [Reiner et al., 2005; Butler et al., 2011]. As mentioned, Desfilis et al. [2018] include this interesting pallial territory of sauropsids instead in their novel caudal dorsolateral pallium (cDLP; Fig. 7a, b) which they homologize with the mammalian perirhinal and lateral entorhinal cortex. Thus, they im-
Fig. 7. a Dorsal schematic view of the brain of the lacertid lizard $P$. algirus at S40 with a colored schematic representation of the pallial divisions postulated by Desfilis et al. [2018], according to a sixpart model, extracted from their Figure 12 (with permission). In the right hemisphere there is a representation of the six divisions seen from the top, while in the left hemisphere there is a representation of the pallial divisions seen more ventrally in a horizontal section through the ventral pallium (VP). Note the small size of the dorsal pallium (DP), and its position topologically comparable to that of the avian hyperpallium. Two novel divisions are present, the dorsolateral pallium (DLP, which includes the lateral cortical superposition that separates DP from LP, plus a small area rostral to it), and the ventrocaudal pallium (VCP, including the dorsolateral amygdala and nucleus sphericus, among other nuclei). The VCP of the lizard is comparable to the avian arcopallium. The olfactory bulbs were left in gray. b Schematic drawings of frontal telencephalic sections of the lacertid lizard $P$. algirus at S38, from rostral (top) to caudal (bottom) levels, extracted from Figure 13 in Desfilis et al. [2018], with permission. The six pallial divisions are distinguished using the same color code used in their Figure 12 and partly reproduced in $\mathbf{a}$. Within each division, the main nuclei and areas are labeled using the classical nomenclature. Note as a discrepancy between the $\mathbf{a}$ and $\mathbf{b}$ maps that in $\mathbf{a}$ the cDLP contacts the VCP, whereas this does not occur in $\mathbf{b}$ (third section level), since cDLP and VCP are separated by the compacted lateral angle of the telencephalic ventricle. c This schema (modified from Fig. 15c, d in Garcia-Calero et al. [2021]) shows on the left a flattened pallium topological map (in mouse) distinguishing the caudally reflected amygdalar pallium (pale yellow) from the larger rostral cortical pallium ( $\mathrm{p}$ is the posterior amygdalo-hippocampal amygdalar portion; the amygdalar field is reflected at a fulcrum at the bottom of the lateral ventricle; check the sagittal section schematized to the right). The cortical pallium is divided into a central neocortex island ( $\mathrm{NCx}$, white) surrounded by a thin mesocortical inner ring (MCx; light grey) and a partly broad allocortical outer ring with olfactory, entorhinal/schizocortical, and hippocampal subregions (OACx, SchCx, HiACx; green). The thick violet colored line alongside the OACx symbolizes the antihem organizer $(\mathrm{AH})$ at the pallio-subpallial boundary, whereas the thick blue colored line alongside the HiACx represents the cortical hem organizer $(\mathrm{CH})$ found along the fimbrial chorioidal taenia. The complementary drawing tt the right of $\mathbf{c}$ shows a schematic sagittal section through the telencephalon which passes along the black straight line included in the previous schema; the section passes through both the olfactory bulb and the pallial amygdala (pale yellow; its posterior amygdalohippocampal nucleus is shown at $\mathrm{p}$ ) and represents the central $\mathrm{NCx}$ island, the surrounding mesocortical ring ( $\mathrm{MCx}$ ), and sectioned rostral and caudal parts of the peripheral allocorticalschizocortical ring ( $\mathrm{OACx}, \mathrm{SchCx})$. The lateral ventricle appears filled in black, and reflects the subpallial intraventricular bulge (Subpall.). This topologic cortical map reproduces notions reported in Puelles et al. [2019a] about a concentric-ring model of the cortical pallium, partly inherited from previous literature. As shown on the right, normally the cortex covers topographically the amygdalar pallium, causing the erroneous impression that the pallial amygdala is a cortical derivative. However, on the left we represent its true topological relative position, obtained if the respective ventricular zones are separated and flattened out. The posterior pallial amygdala (p) falls close to the SchCx as well as to the caudal ends of the OACX and HiACx (where both the $\mathrm{AH}$ and $\mathrm{CH}$ finish). 
plicitly assume, I guess, that the mammalian temporal cortex domain does not exist in lizards, or is represented instead (at least in evolutionary potency) within the small rostral patch of the reptilian dorsal pallium (their DP; Fig. 7a, b), as well as within the classic avian Wulst. This interpretation only applies if these authors agree with me that no part of the neocortex arises evolutionarily from the DVR. I will return under point 3 below to apparent defects in the proposed homology of the lizard cDLP with the mammalian entorhinal cortex.

\section{The LP Issue}

The ventral part of the claustro-insular LPall or avian MV (which contains, like the MD, evident $\mathrm{Nr} 4 a 2$-positive claustral and Cyp26b-positive insular elements in the chick; Fig. 4c-f) inexplicably was not ascribed in the lizard to iDLP (like the rest of the claustro-insular complex), but to the small lizard LP. However, Desfilis et al. [2018] did not examine the Nr4a2 and Cyp26b markers in the lizard before proposing this conceptual change. The latter possibly rests exclusively on the absence of $\operatorname{Lh} x 9$ expression in this lizard area (a marker not identifiable in the chick or the mouse). I hold that a conceptual separation of MD/ $\mathrm{MV}$ is not realistic because we showed in the chick that they subdivide gradually out of a primarily unitary mesopallium primordium (e.g., Fig. 3c; Puelles et al. [2016a]; see also relevant Briscoe and Ragsdale [2018] molecular data). In our developmental analysis of the chick claustroinsular complex we interpreted the objective process of $\mathrm{MD} / \mathrm{MV}$ division, the differential histogenetic and molecular development of which we actually first described step by step [Puelles et al., 2016a], as a secondary dorsoventral subdivision of the claustro-insular complex (LPall). This is topologically comparable to the secondary differentiation of mammalian disgranular and agranular insular subareas, both of which have distinct deep claustral components [Puelles, 2014; Fang et al., 2021).

The proposed lizard LP also includes a lateral part of the olfactory cortex, with its longitudinal dimension restricted to this small LP sector (this obviously is inconsistent with our postulate that all of the olfactory cortex belongs to VPall; compare Fig. 2, 4e, f). Such a rostrocaudally restricted piriform cortex subdivision is not known to exist in mammals, birds, or reptiles. Moreover, Desfilis et al. [2018] arbitrarily ascribe the lizard EPd homolog appearing under the lateral olfactory cortex to their "LP," without giving specific supporting data that would correct my evidence of its tangential migrated origin from the claustral part of LPall, or employing appropriate selective markers (EPd was found to be selectively Nr4a2- positive and derived by tangential migration from the primordial LPall claustrum population in the chick and mouse by Puelles [2014], Puelles et al. [2016a], Puelles [2017], and Watson and Puelles [2017]). I thus think that none of the three components of the proposed lizard LP is convincingly defined. What Desfilis et al. [2018] call "LP" clearly belongs rather to our VPall, since it contains both the piriform cortex and the migrated claustrum-derived EPd.

\section{The DLP Issue}

The newly postulated lizard DLP pallial sector is an extensive longitudinal territory found intercalated between the lizard MP and VP, except at relatively rostral "intermediate" levels, where it appears sandwiched between the small DP and LP domains (Fig. 7a, b) [Desfilis et al., 2018]. Rostral, intermediate, and caudal DLP parts are distinguished (rDLP, iDLP, cDLP). The small rDLP found near the stalk of the olfactory bulb ends rostrally short of the rostral MP/VP transition. The iDLP relates to DP and LP and represents the avian MD (upper half of my claustroinsular LPall) plus neighboring HI/HD hyperpallial regions [Puelles et al., 2016a]. The large cDLP reaches the caudal MP/VP transition, but without contacting the amygdalar pallial field (including VCP), from which it is separated by the olfactory cortex (VP; Fig. 7b); this relationship is incorrectly represented in Figure 7a, since VCP clearly cannot be seen in a dorsal view (see legends). The cDLP is held to represent the mammalian perirhinal and entorhinal cortex.

The reptilian DLP includes the area of the classical lateral cortical superposition where a double cortical plate exists, olfactory projections are received marginally, and abundant superficial reelin-expressing neurons are observed [Goffinet et al., 1999; Puelles et al., 2017]. Desfilis et al. [2018] further think that the DLP is a unique site displaying inside-out cortical layering, although there exist contradictory lizard neurogenetic data (Goffinet et al. [1986]; see their Fig. 2a); the existence of a reptilian outside-in pallial layering particularly in this area was discussed in Puelles et al. [2016a]. Much of the molecular evidence presented to identify the different parts of the DLP sector in the lizard centers on shared expression of Lhx9. However, the authors acknowledge that a topologically comparable expression pattern of $L h x 9$ does not exist in either the chick or the mouse pallium (their Table 2 and text). The use of this marker to define pallial sector boundaries is thus not validated comparatively, particularly in the absence of any corroborating analysis of $\mathrm{Nr} 4 \mathrm{a} 2$ and Cyp26b expression patterns. This leaves mainly topo- 
logical considerations of relative positions and the weight we may be disposed to give to connectivity data for judging the homologies proposed. I prefer to leave aside the connections until we are sure of the regional homologies.

In the comparisons of DLP parts with hypothetically corresponding mammalian structures (Desfilis et al. [2018]; their Table 1 and Discussion), we learn that the homolog of the lizard rDLP would be found at the orbitofrontal cortex and associated dorsal peduncular cortex. It is possible that reference is made here to the transitional orbitary mesocortex, rather than to the dorsopallial orbitofrontal neocortex (see Puelles et al. [2019a] and below).

The neighboring iDLP is not identical to our LPall since it incompletely represents both the mouse insular cortex and the underlying claustrum (i.e., only the avian MD part, excluding MV). The MD portion is characterized in birds by selective expression of $C b \ln 2$, also not studied in this work [Reiner et al., 2011; Puelles et al., 2016a]. Moreover, within their iDLP Desfilis et al. [2018] include a lateral part of my DPall (namely the NI/ND hyperpallium areas of birds; incidentally, this doubtful ascription might be another viewpoint relevant for discussing the Atoji et al. [2018] data on NI/ND connectivity). It is true that these areas have been shown to share numerous markers with the classic MD/MV complex (we pointed this out in Fig. 20 of Puelles et al. [2017]), leading to other proposals to include HI/HD within the mesopallium [Jarvis et al., 2013; Briscoe and Ragsdale, 2018]. However, the NI/ND complex demonstrably remains distinct cytoarchitectonically from $\mathrm{MD} / \mathrm{MV}$, and does not express either Nr4a2 or $C y p 26 b$ in the chick, even after claustral cells migrating into the apical hyperpallium have passed subpially through them [Puelles et al., 2016a; Puelles, 2017]. HI and HD do pose a comparative problem that has not yet been addressed satisfactorily; in Puelles et al. [2016a] we conjectured that they might jointly correspond to the peculiar oral-related ventral parietal cortex area distinguished in the mammalian DPall (ibid; VPa in our Fig. 12); alternatively, they might correspond to the granular insular area, if we assume that it actually falls within DPall, as is perhaps suggested by its granularity, leaving only disgranular and agranular insular areas in the mesocortical LPall. There are so far no conclusive data about these hypotheses. I must note that, remarkably, Atoji et al. [2018] did not propose any mammalian homologies for the avian HI/HD, irrespective of having obtained their hodological data.

The true mammalian LPall also includes caudally the structurally and molecularly comparable PRh cortex (see Puelles et al. [2019a]), interpreted instead to be included

Sauropsidian Claustro-Insular Homology within the controversial claustro-insular iDLP notion of Desfilis et al. [2018]. Leaving aside the "caudal insular" $\mathrm{PRh}$ cortex domain, I will assume for the sake of argument that the lizard cDLP might wholly correspond according to Desfilis et al. [2018] to the lateral entorhinal cortex (ERhL). The cDPL roughly correlates topologically with our avian $\mathrm{HC}$, to which I believe it may be really equivalent (it would thus be rather a caudal part of the DPall, in my opinion [see Fig. 12 in Puelles et al., 2016a], but not an entorhinal cortex - which I ascribe instead to the allocortical schizocortex in Puelles et al. [2019a]). I hold that the entorhinal cortex homology hypothesis for the lizard cDLP is not possible, for reasons detailed next.

In Puelles et al. [2019a] we showed that the mammalian entorhinal cortex (both lateral and medial) forms part topologically of the outer allocortical ring around the inner mesocortical ring that encloses the central neocortex island. The mesocortex ring includes laterally the claustro-insulo-perirhinal complex, followed caudally by the postrhinal cortex, which connects dorsomedially with the cingular cortex. The latter meets rostrally the dorsal peduncular cortex and the posterior orbital cortex; the latter closes the mesocortical ring when it meets the rostral insula. The rodent allocortical ring is wide laterally (piriform cortex) and caudally (entorhinal cortex and invaginated hippocampus) but dwindles into the thin indusium griseum and taenia tecta domains medially and rostrally, the last of which reaches the olfactory stalk area, thus closing the outer cortical ring [Puelles et al., 2019a]. It is usually accepted that the non-mammalian hippocampus also has equivalent rostromedial supra- and precommissural portions, irrespective that there is no associated corpus callosum (discussion in Puelles et al. [2019b]); incidentally, this is another fundamental hodological difference in the pallium of tetrapods that apparently shatters no homologies (compare my comments above on Atoji et al. [2018]).

Basically, the outer cortical ring comprises the threelayered (allocortical) olfactory and hippocampal cortex, but it also contains the intercalated and more complex parahippocampal schizocortex domain. The latter is represented by the presubiculum, parasubiculum, medial entorhinal cortex and lateral entorhinal cortex, all of which possess an additional superficial stratum that becomes more complex along the sequence of areas cited above. The topologic position of the schizocortex strictly within the outer cortical ring (as illustrated with Encl data in an advanced mouse embryo in Puelles et al. [2019a]) is corroborated by its demonstrable direct contact via the amygdalo-piriform transition area with the neighboring 
but non-cortical pallial amygdala field (Garcia-Calero et al. [2020, 2021]; Fig. 15c, d in the 2021 reference). Furthermore, there is no doubt that the mammalian entorhinal schizocortex fully separates the piriform cortex from the hippocampal cortex (additional literature is cited and illustrated schematically in Puelles et al. [2019a]). It can be discussed whether the evolutionary emergence in mammals of the schizocortical structural singularity within the three-layered allocortex ring is due to evolutive modification of either the olfactory or hippocampal cortex, or perhaps of both of them (producing the ERhL/ ERhM distinction, as was suggested by Abellán et al. [2014]). Alternatively, the schizocortex might represent a primary Bauplan feature evolved from an initially molecularly distinct three-layered intercalated part of the allocortex already present between Pir and Hi in the ancestors of mammals and sauropsids, which evolved differentially to its Pir and Hi neighbors, generating SchCx (I rather like this possibility).

A flat map comparison (Fig. 7a, c) shows that this intimate topological relationship of the mammalian entorhinal schizocortex with olfactory and hippocampal allocortex, as well as with the pallial amygdala, does not exist between the CDLP and the simpler lizard ring formed only by olfactory VP and hippocampal and parahippocampal MP as mapped by Desfilis et al. [2018] (their Fig. 12,13 , reproduced here as Fig. 7a, b). Specifically, the caudal part of cDLP (their ERhL candidate) lies concentrically (i.e., inside) the simplified outer allocortical ring formed by olfactory and hippocampal areas. The caudal DLP is actually portrayed as "parallel" lengthwise to the MP and VP moieties of that ring. This position of the purported ERhL cortex homolog is therefore topologically wholly inconsistent with the corresponding mammalian arrangement of the schizocortex. Importantly, the mapped locus of caudal cDLP inside the simplified allocortex ring impedes any possible contact with the supposedly homologous pallial amygdala, which is found topologically outside the reptilian, avian, and mammalian allocortical pallial ring (Fig. 7b; note Fig. 7a falsely represents the VCP amygdalar component as if it were visible at the dorsal cortical surface, touching the $\mathrm{CDLP}$, a relationship that Fig. 7b shows is impossible). However, an ERhamygdala mutual contact relationship is readily observable in mammals, irrespective that the amygdala is tucked morphogenetically underneath the caudal border of the cortical field (Fig. 7c). There is accordingly no topologic correspondence of the lizard cDLP as defined by Desfilis et al. [2018] with the mammalian entorhinal cortex. In my opinion, this makes their homology hypothesis unten- able. The true lizard ERh homolog must be a part of the allocortical ring. This feature maybe redirects the field's attention to our proposal that the distinct cDLP pallial region observed in reptiles and birds may be topologically homologous instead to the caudal temporal neocortex, being thus a formerly unrecognized part of the sauropsidian DPall [Puelles et al., 2016a, 2017].

A separate issue is whether the sauropsidian LPall is continuous rostrally and caudally with other cortical domains, as seen in the mouse [Pattabiraman et al., 2014; Puelles et al. 2019a]. This notion is indeed suggested on different grounds by Desfilis et al. [2018], as it was previously by Dart [1934]. In this sense what we possibly have in the lizard DLP and the hexapartite pallium model is an incomplete idea of a reptilian concentric ring model, which we might come closer to in the future. The postulated lizard DLP domain already extends rostrally and caudally from the claustro-insular intermediate site, and includes perhaps correctly the perirhinal cortex, but is not related yet to a ring-like complete configuration around the DP, lacking postrhinal and cingulate components.

The future-oriented conceptual alternative offered in the concentric ring model of the mammalian pallium seems advantageous in terms of easier causal explanation by peripheral organizers and sources of migrating CajalRetzius neurons (see antihem and cortical hem mapped in Fig. 7c; reviewed in Borello and Pierani [2010], Puelles [2011], Arai and Pierani [2014]). In my opinion this model supersedes the now probably outdated notion of parallel longitudinal pallial domains extending from pole to pole, as contemplated controversially before by us and others (i.e., pallial sectors extending uninterrupted across both cortex and amygdala; see Fig. 7a, b). The concentric ring model admits a different (circular) sort of "sequential" regionalized similarity of some classical cortical regions (mesocortical, allocortical, and schizocortical), emphasizing their concentric peripheral versus central boundaries, while allowing differential local arealization and a simpler approach to differential patterning via several external "cortical rim" secondary organizers and migrated Cajal-Retzius "messengers."

In my opinion the analysis of the claustro-insular region of the lizard offered by Desfilis et al. [2018] (see also Medina et al. [2019]) is not well supported in its variant details but perhaps will be corrected in the future with more appropriate data, perhaps coming closer to the cortical-ring model. It must be pointed out that the sauropsidian mesopallium embodying the claustro-insular homolog candidate represents a well delimited and molecularly distinct pallial sector that was curiously left aside by 
Karten [e.g., 1969, 2015] and his followers [e.g., Reiner et al., 2005; Butler et al., 2011], but has been fully corroborated as a molecularly distinct pallial module (or "dedicated part") in several recent approaches [Puelles et al., 2017; Briscoe et al., 2018; Tosches et al., 2018]. Surprisingly, it actually represents the first mammalian cortical region whose homology in sauropsids seems strongly established according to all primary criteria of homology. I thus logically oppose its disguise in reptiles as something else than the mesocortical LPall we first postulated $[\mathrm{Pu}-$ elles, 2014; Puelles et al., 2017] and Tosches et al. [2018] demonstrated.

In addition to the already mentioned novel DLP pallial sector, Desfilis et al. [2018] also postulated another new pallial sector called the ventrocaudal pallium (VCP). This VCP domain strictly represents a subdivision of the pallial amygdala. Indeed, Desfilis et al. [2018] still assumed, as many of us initially did, that the cortical palli$u m$ includes the pallial amygdala. However, in recent amygdala work we have strongly argued against this notion, and we presently defend a separate amygdalar pallium placed topologically ventral to the cortical pallium [Puelles et al., 2019a; Garcia-Calero et al., 2020, 2021; Garcia-Calero and Puelles, 2021]. These two major pallial domains are presumably patterned separately and differentially, though admitting a number of shared genes, which led us all to error [e.g., Medina et al., 2004]. Note that the cortical hem and antihem organizers do not seem to act upon the amygdala (see Garcia-Calero et al. [2021]; their Fig. 15c, d are reproduced here in Fig. 7c). Accordingly, good models of the cortical pallium should not have parts in the amygdalar pallium, which is a reason for leaving the VCP of Desfilis et al. [2018] out of the present discussion. The claustro-insular complex instead clearly belongs strictly to the cortical pallial field and is not represented at all within the amygdalar pallial field [Puelles, 2014; Puelles et al., 2019a].

\section{M.A. Tosches and Colleagues}

A breath of new air arrived with a report by Tosches et al. [2018] on single-cell transcriptomics of reptilian pallium (turtle and gecko lizard brains) with various computed correlations with available mouse and human data. The natural location of the main cell types discovered was mapped by in situ hybridization. These authors strongly support a reptilian claustrum homolog.

Several important results were derived from this analysis:

(1) Conventional (classic) M, DM, and posterior D pallium $(\mathrm{pD})$ regions showed molecular profiles largely coinciding with the mammalian hippocampal cortex. The $\mathrm{M}$ cortex correlated (as expected) with dentate gyrus profiles, whereas the DM and $\mathrm{pD}$ cortex correlated with CA3 and CA1 field profiles, roughly in mediolateral positional order.

(2) The anterior $\mathrm{D}(\mathrm{aD})$ cortex correlated with the mammalian neocortex, showing glutamatergic cell types with either mixed layer $2 / 3 / 4 / 5$ a characteristics (individual clusters showing different mediolateral or rostrocaudal locations, indicating a degree of cortical arealization) or layer $5 b / 6$ properties (independently of areal subregions). The lower layer-like cells occupy superficial positions, compared with upper layer-like cells found deep to them (outside-in layering, as found previously by Goffinet et al. [1986]; comparable also to the avian pattern).

(3) The reptilian anterior and posterior parts of the dorsal ventricular ridge (DVR) produce distinct cell clusters comparable to amygdalar pallial neurons (this clearly supports our thesis of a separate amygdalar pallium, as well as about a lack of homology of the sauropsidian DVR with mammalian cortical pallial regions, contrary to the speculation of Karten [1969]). Specifically, the aDVR compares with the lateral amygdalar nucleus in its cell typology, whereas the pDVR compares to the rest of the pallial amygdala (basolateral, basomedial, cortical, and amygdalo-hippocampal nuclei; see our recent radial amygdalar model, which visualizes these nuclei as components of radial histogenetic units; Garcia-Calero et al. [2020]). Although numerous effector (function-related) genes found expressed in particular regions of the aDVR are shared with those in the $\mathrm{aD}$ cortex clusters (with neocortex-like cells), the authors argued that this does not reveal homology, because selective analysis of transcription factors (TFs) involved in fundamental fate specification shows differential profiles. The shared (functional) effector gene clusters therefore can be explained as convergently recruited elements related to evolved functional analogy (e.g., analysis of diverse known sensory inputs to the DVR, which mimic parallel sensory input to the neocortex-like $\mathrm{aD}$ cortex). This analysis of DVR versus cortical transcriptomics represents strong evidence against those still defending the speculation of Karten [1969] on the homology of sauropsidian DVR cell types with those in given layers of the mammalian neocortex, even though the predicted mammalian migrations (or pallial sector duplication) had remained undemonstrated (Reiner et al. [2005]; see also the same critique in Medina et al. [2013] and comments in Puelles et al. [2007, 2017, 2019b]). The notion that sauropsidian DVR might be largely homologous to mammalian amygdala was postu- 
lated previously by Bruce and Neary [1995], Bruce [2007, 2012], and Puelles et al. [2007, 2019b].

(4) Importantly for the present commentary, the pallial thickening domain of the turtle T. scripta elegans (an incipiently invaginated part of the rostrolateral pallium, distinct from the DVR proper; see Puelles et al. [2017]) showed unique cell clusters corresponding in molecular profile to the mammalian claustrum. Four markers uniquely identified this region in in situ mappings (Lgals1, Adarb2, Pou3fa, Crhbp) and 14 genes known to characterize the mouse claustrum (including Nr4a2, Gng2 and Ctgf) were correlated with the same cell clusters. These findings were further corroborated in the lizard Pogona vitticeps, where, instead of forming a pallial thickening, the claustrum-homolog population appears largely invaginated, forming the rostromedial part of the DVR, similarly as occurs with the avian mesopallium. As expected, this pallial domain of Pogona retains a rostrolateral-free pial surface, like the avian topological and molecular LPall homolog.

These results fully corroborate our deductions on a plausible claustro-insular homolog primordium in several reptiles (turtle, lizard, snake) [Puelles et al., 2017] and our previous conclusions on the avian and turtle claustrum [Puelles et al., 2016a; Puelles 2017]. This coincidence is not totally unconnected, since during the preparation of the Tosches et al. [2018] publication (and while I was preparing the Puelles et al. [2017] chapter) I repeatedly met M.A. Tosches and some of her colleagues at various scientific meetings and we discussed where a claustrum homolog might be possibly found in the species they were studying. Previous anatomic studies of the reptilian telencephalon had largely missed the rostrolateral claustral territory due to the risky habit of studying and representing pallial structure in a single cross-section taken at mid-levels of the hemisphere (sometimes this implied falsely representing the rostral pallial thickening at such mid-level sections). This habit also subliminally introduced the false assumption that pallial domains tend to stretch longitudinally along the whole area map of the pallium (e.g., as in the model of Desfilis et al. [2018]), a viewpoint now opposed by the concentric-ring model [Puelles et al. 2019a, b].

Further evidence that has accrued recently on the lizard claustrum homolog was functional in nature and was reported by Norimoto et al. [2020]. These authors (from G. Laurent's lab, the same producing the work reported by Tosches et al. [2018]) performed physiological studies on the lizard Pogona vitticeps claustral domain. These showed that this pallium region (previously characterized transcriptomically as a claustrum homolog in this species by Tosches et al. [2018]) promotes functional transition of the dorsopallial cortex (anterior D pallial area) into slow-wave sleep phase via excitatory activation of cortical inhibitory neurons. It may be surmised that ulterior cortical efferent activation of claustral inhibitory interneurons ends the slow-wave sleep phase. This result weighs significantly against the negative evidence on claustrocortical connections advanced by Atoji et al. [2018], as commented above. This line of research apparently began with the previous finding of Shein-Idelson et al. [2016] (same lab again) that this lizard displays slow-wave and REM sleep phases during its short (3-min long) periods of sleep. Independently, as far as I know, a Japanese group led by Y. Yoshihara demonstrated the same slow-wave sleep-inducing function for the mouse claustrum [Narikiyo et al., 2020; Yoshihara 2021].

These marvelous functional results surpass in an unexpected way the earlier functional conjectures previously collected in the book The Claustrum [Smythies et al., 2014], where my developmental contribution [Puelles, $2014]$ is contained. The cited progress associating the mammalian and reptilian molecularly defined claustrum homologs (and thus presumably also the avian one) via claustro-cortical connections with a fundamental brain function such as slow-wave sleep is fascinating. One may then ask: when did slow-wave sleep emerge in evolution? Was such sleep present in the earliest vertebrates, or was it acquired subsequently? That is, do all vertebrates need a claustrum homolog? With regard to a pragmatic division of comparative neuroanatomists into those emphasizing conserved homologous aspects just varying emergently in aspect, connections, and functions, and those emphasizing step-like evolutionary emergence of key structural and functional innovations, I belong to the former (see comments in Striedter and Northcutt [2020]).

A partial deficiency I spotted in these recent lizard and mouse studies, starting with Tosches et al. [2018], is that they leave aside the possible insular cortex homolog alluded to in Puelles et al. [2016a], Puelles [2017], and Puelles et al. [2017]. This is understandable in the case of Narikiyo et al. [2020] because their technical approach in the mouse involved selectively the claustrum cell population. Norimoto et al. [2020] instead seem to identify the whole LPall sector as "claustrum." It should be remembered that the mammalian claustrum is only the earlyborn component of the mammalian LPall, the later-born component being the subsequently produced insularperirhinal cortex layers [Puelles, 2014]. One would expect the demonstration with appropriate markers and single 
cell transcriptomic analysis that any studied non-mammal only has a claustrum-like cell population in its LPall, excluding any insular cell population. This is theoretically possible, even if improbable, but has not been demonstrated so far in the sauropsids studied. Lacking such specific evidence, the LPall pallial sector must be regarded as an indissoluble field-homologous claustro-insular histogenetic unit (a fundamental morphogenetic unit or FMU sensu; Nieuwenhuys and Puelles [2016], Nieuwenhuys [2017]). I expect that its evolution proceeds as a whole given that a single progenitor domain is involved, so that both claustral and insular cells should be found, maybe with changing relative distributions (see the possible $C y$ p26b-labeled "insular cells" in the chicken compared to those in the mouse; Fig.4; Puelles [2017]).

We do not have any evidence yet of a hypothetic ancestral state in which this progenitor domain produces a claustrum homolog devoid of an insular complement. The LPall can thus be regarded either as a field-homologous undifferentiated claustro-insular whole (as it may exist, say, in cyclostomes, or generally in anamniotes), or as the sum of two one-to-one homologs produced developmentally in series, one after the other: the claustrum and the insula. The latter option seems to be the case in sauropsids insofar as the claustral gene markers so far mapped in these species (e.g., Nr4a2) largely label positionally restricted claustral cell populations within the LPall region representing the early-born populations. This means that bona fide sauropsidian one-to-one claustrum homologs already have been identified by topological, developmental, and special (molecular; transcriptomic; functional) criteria. It should therefore be deduced that the remaining non-claustral LPall cells correspond to a presumptive insular field-homolog (since there are no separate layers) plus inhibitory interneurons imported from the subpallium. This conclusion is clearly supported by our preliminary chicken data using the mouse selective insular marker Cyp26b (Fig. 4) [Puelles, 2017; Puelles et al., 2017]. I accordingly think it is misleading to describe the whole LPall histogenetic unit as "the claustrum" [e.g., Norimoto et al., 2020], or to suggest that the insular cortex somehow falls within DPall or DLPall categories [Wullimann, 2017a, b]. Desfilis et al. [2018] did not state their position on this aspect.

\section{S.D. Briscoe and C.W. Ragsdale}

Another recent work I need to comment upon with regard to our sauropsidian-mammalian claustrum homology hypothesis is the report of Briscoe et al. [2018] coming from Ragsdale's lab - on differential molecular

Sauropsidian Claustro-Insular Homology characteristics and possible homologies of the sauropsidian mesopallium (= our LPall and claustro-insular homo$\log$ ). They cite the Puelles et al. [2016a] report in their introduction as a work that supports a "claustral" nature of the avian mesopallium, but do not discuss this hypothesis in their discussion at all. Importantly, they do not compare their data (particularly those on Nr4a2) with ours, nor with the mouse claustro-insular complex. It is difficult to believe that knowing about our hypothesis they did not critically compare their results with ours. Their published positions on pallial homologies are opposed in principle to ours (see also other parallel publications: Dugas-Ford and Ragsdale [2015], Briscoe and Ragsdale [2018, 2019]). Their striking silence in this regard thus invites interpretation. I suppose that their silence means that they did not find any evidence contrary to our hypothesis, but why not say so?

These authors usually support Karten's [1969] speculation on the essential "neocortical" nature of the sauropsidian dorsal ventricular ridge (DVR), an idea I have criticized on several grounds, as was mentioned above and will be elaborated below [Puelles et al., 2007, 2017, 2019b]. We are thus openly scientific opponents. It accordingly was to be expected that this report focusing specifically on the mesopallium would examine any weak points in our previous analysis of Nr4a2 and Cyp26b [Puelles et al., 2016a, 2017; Puelles, 2017] in the light of the ampler list of gene markers used by these authors.

In the Introduction, after commenting a la mode that birds are intelligent and perform cognitive tasks comparable to those of mammals, Briscoe et al. [2018] start their argument as follows (my italics):

"Neither birds nor non-avian reptiles possess a six-layered neocortex, the neuroanatomical hallmark of all extant mammals. In place of a laminar structure, the bird dorsal telencephalon (DT) contains territories of clustered neuronal cell bodies, or nuclei.... The bird DT is organized into two main divisions, the Wulst and the dorsal ventricular ridge (DVR).... The DVR is further subdivided into the mesopallium, nidopallium, and arcopallium."

This "dorsal telencephalon" concept (DT) does not refer to a part of these brains that is well known among experts but to a novel construct of these authors; it is a lumped way of looking at the pallium. This term puts together under the DT notion the very different "Wulst and DVR" pallial domains (remember the conclusions of Tosches et al. [2018]). I interpret it as presenting subliminally the hidden conclusion that Wulst and DVR jointly represent the homolog of the mammalian cortex. The latter concept as used by these authors apparently is also a 
mammalian DT that lumps all pallial parts distinguished habitually (neocortex, mesocortex, allocortex, pallial amygdala), except the hippocampal cortex (the sauropsidian DT also leaves out only the hippocampal pallium). Briscoe et al. [2018] thus dismiss all finer anatomic and developmental subdivisions recorded in the literature and would be giving us as a preconceived fact, hidden under the novel term DT, that the dual Wulst+DVR aggregate is a unitary avian pallial region comparable as a whole to an equally lumped mammalian pseudo-neocortex. The rationale for this clearly anti-anatomic operation is not presented, but we will see to what it leads.

This hidden assumption at the start can be recognized as a novel form of the theory defended by Karten's school (e.g., Karten [1969] and his subsequent publications up to Karten [2015]; Reiner et al. [2005] and Butler et al. [2011]). This theory is followed explicitly by the present authors in Dugas-Ford and Ragsdale [2015] and Briscoe and Ragsdale [2018, 2019], and is unilaterally considered by them to supersede the allegedly old and unnecessary anatomical pallial subdivision complexities supposedly indulged in by unmentioned contemporaries. The invention of the unified "dorsal telencephalon" concept is however truly their own addition to Karten's theory (somewhere they speak of offering a "strong version" of the same). They possibly may have followed the thought that earlier separate homologizing of Wulst and DVR with different parts of the neocortex by Karten and followers implied belief in a hardly credible dual evolutionary origin of the neocortex (e.g., Butler et al. [2011]; this was one of the criticisms I recorded in print). By lumping together the Wulst and DVR into an assumed unitary "DT" concept it was possibly thought that the dual cortical origin difficulty disappears.

For newcomers to the field I should explain that Karten's DVR/neocortex theory is based on a set of hodological data in sauropsids (now more than 40 years old) interpreted originally by Karten [1969] as indicating that separate sauropsidian DVR cell populations, irrespective of their radically different developmental positional origins, are similar and virtually "the same" (i.e., homo$\log$ ) in nature, connections and function as given mammalian neocortical cell populations corresponding to various cortical regions and specific layers within them (fuller review and discussion in Puelles et al. [2007, 2019b]). Remarkably, a second parallel Wulst/neocortex homology hypothesis of Karten [1969] is actually not objectionable (and has not been objected to by anybody), except in its reference to only a part of the mammalian neocortex whose precise borders have never been determined. It re- fers to direct comparison of dorsopallial (DPall) sauropsidian and mammalian progenitor areas. This is a case of homotopic Bauplan comparison, though the whole avian DPall is compared to only an undefined part of the mammalian DPall. The DVR/neocortex comparison instead refers to topologically different progenitor areas, since the sauropsidian DVR encompasses the VPall and LPall sectors but not the DPall, where the mammalian neocortex resides. This hypothesis is accordingly opposed by different contemporaneous authors including me, because we do not admit different topologic origins within the pallial Bauplan as a basis for homology, irrespective of any adduced "feature similarities" such as connections (these automatically fall under the concept of analogy due to their differential topologic positions; Nieuwenhuys [2017], Nieuwenhuys and Puelles [2016], Puelles and Medina [2002], Kuhlenbeck [1967], Remane [1952], Jacobshagen [1925, 1927], Owen [1848]).

Karten [1969] initially offered an acknowledged speculation (as stated in his title) to make credible his DVR/ neocortex homology proposal. His argument was that, theoretically, mammalian pallial populations "cell-homologous" (meaning hodologically similar) to sauropsidian ancestral DVR cell populations might migrate developmentally (in mammals) out of positionally DVR-like embryonic sites (e.g., the mammalian VPall) into appropriate places of the developing mammalian dorsopallial neocortex (a consistent embryologic origin was thus thought to solve the topologic disparity problem, but this requires that the postulated migrations be demonstrated, and this has not happened so far; Karten and colleagues never worked on mammalian embryos to check this point; those mammalian tangential migrations that have been discovered by others do not show the properties demanded by Karten's speculation; see Puelles [2011]). The hypothetic multiple migratory process only occurring in mammals was held not to impede the diverse migrated cell populations to reproduce their original ancestral sauropsidian DVR connections (no explanation was ever offered detailing how this was achieved; see comments above on Atoji et al. [2018]). Guidance of the postulated migrations to distinct neocortical sites and layers was never explained, either, and no mechanisms were suggested for how observed uniform neocortical neurogenetic gradients are obtained out of cell populations derived from several separate origins (Wulst versus various DVR sites). Such a non-parsimonious argument with undemonstrated assumptions since 1969 seems to me to be simply not acceptable. I discussed personally these points many times with H. Karten himself in the early 1990s 
(during a stay in La Jolla), in the attentive presence of R.G. Northcutt. My doubts were expressed in Puelles et al. [2000], Puelles [2011], and Puelles et al. [2007, 2019b]. I should make clear that I have the highest personal respect, admiration, and friendly affection for Harvey Karten, with whom I remain in contact. He did much in science that is not subject to any criticism.

As said, the predicted pallio-pallial migrations never appeared in mammalian embryos, though other migrations into the neocortex (subpallio-pallial ones and even some pallio-pallial ones) were studied or commented on by independent authors [e.g., Puelles, 2011, 2014]. As a result of the accumulated evidence that the original migratory speculation does not really support Karten's $\mathrm{DVR} /$ neocortex homology, the persistent believers in Karten's DVR homology hypothesis (based now nakedly on 'Bauplan-free' cell populations) have ceased in recent times to postulate any migrations, referring instead vaguely to "duplicated" DVR progenitor domains (see Reiner et al. [2005]; Butler et al. [2011]; however, no evidence, explanation, or mechanism for the speculated "duplication" was advanced either). In most cases these are speculations about what might happen during mammalian development or evolution presented by scientists that do not work with mammalian materials.

The work of Suzuki et al. [2012] and Suzuki and Hirata $[2013,2014]$ actually follows a slightly different line of argumentation on dual progenitor origins of the mammalian neocortex in comparison to the possible homolog populations in sauropsids (cells supposedly homologous to cortical deep layer cells were held to arise in sauropsids at different loci than cells homologous to upper layer neurons). This thesis is sometimes evaluated as supporting Karten's DVR/neocortex homology hypothesis. I discussed personally with Suzuki and Hirata in Japan, and also separately with Hirata in Oxford, without reaching a consensus. See my critical comments on their ideas in $\mathrm{Pu}$ elles et al. [2016a, 2017]. E.D. Jarvis and C.C. Chen were present at the same table in Japan but they did not really participate in the discussion. This was shortly before the Jarvis et al. [2013] and Chen et al. [2013] reports appeared (preliminary results were presented in that meeting). I later recorded detailed critical comments on some aspects I considered highly aberrant in these two papers [Puelles et al., 2017] and there are recent reactions on their side in Gedman et al. [2021] and Biegler et al. [2021] (see below).

After this possibly needed excursus on Karten's speculative theory on dual neocortex origin, I would like to point out that Karten's DVR/neocortex thesis (used as a dogma by Briscoe and Ragsdale) has turned out to be in-

Sauropsidian Claustro-Insular Homology consistent with the Tosches et al. [2018] single cell transcriptomic analysis of turtle and lizard DVR versus cortical primordia. These data indicated that the VPall part of the anterior DVR (the nidopallium) is possibly analogous to neocortex in some functional synaptic aspects (e.g., processing of afferent thalamic input signals) but is definitely not molecularly regionally homologous to the neocortex in terms of combinations of expressed TFs. VPall seems homologous instead (in this restricted sense) to the lateral amygdalar nucleus. The posterior DVR (the arcopallium) molecularly resembles the rest of the pallial amygdala. Similar amygdalar comparisons were suggested previously on the basis of connections by Bruce [2007, 2012]. Additionally, the LPall portion of the sauropsidian DVR (the mesopallium) was shown by Tosches et al. [2018] to be molecularly and topologically homologous to the mammalian claustro-insular cortical complex [ $\mathrm{Pu}-$ elles et al., 2016a; Puelles, 2017]. This is not a part of the mammalian neocortex but of the transitional mesocortex [Puelles et al., 2019a]; the Ragsdale lab nevertheless lumps it in practice into the neocortex, arbitrarily, by dint of their semantic introduction of the unifying "dorsal telencephalon" notion. The work of Tosches et al. [2018] is also important because it shows that cortical cell types and positions are not constant or even "similar" between reptiles and mammals as is often pretended. I have argued that the same possibly occurs with circuitry [Puelles et al., 2017].

Briscoe et al. [2018] did not cite or discuss the Tosches et al. [2018] report. They nevertheless did cite Naumann et al. [2015], Naumann and Laurent [2017], and Tosches et al. [2018] in their more recent review on the reptilian telencephalon [Briscoe and Ragsdale, 2019]. The answer of Briscoe and Ragsdale [2019] to this epochal transcriptomic work is as follows (my italics and brackets):

"One recent study (Tosches et al., 2018) noted that the pallial excitatory neurons in reptiles and mammals differ in other molecular properties [obliquely referring to transcription factors] and concluded that the cells are therefore not homologous. However, it is similarity, and not dissimilarity, that forms the basis for judgments of homology. Non-homologous molecular features must be permitted, and even expected, to coexist with homologous [meaning similar] features in different amniote pallia given their divergent architectures and 320 million years of independent evolution ...."

This unintelligible position (at least for me) apparently denies the substantial contrary evidence presented by Tosches et al. [2018] to their main thesis: "sauropsidian DT is homologous to mammalian DT." Briscoe and Rags- 
dale [2019] apparently present their conclusions as supported both by the similarities they emphasize and the non-similarities that some strong data of other sources have recently pointed out, which are in essence inconsistent with the preconceived "similarity" conclusions. Unfortunately for Briscoe and Ragsdale, there are no such unassailable positions.

Let us have a look at their homology rationale as expressed in Briscoe and Ragsdale [2019] (my italics):

"In order to understand the evolutionary origins of the mammalian neocortex, it is necessary to identify homologous neocortical features shared with sauropsids. The problem is that homology is based upon similarity, and evolution has rendered the amniote pallial structures into utterly dissimilar forms."

This dissatisfaction with dissimilar forms apparently leads them to leave aside all detailed anatomic subdivisions, formanalytic approaches, and molecular-sector models, including of course mine, in their "dorsal telencephalon" concept.

Elsewhere in the same text we find: "Comparisons made at the levels of circuitry and cell types, rather than neuroanatomical structure, have provided a wealth of insight into amniote pallium evolution."

They clearly negate precisely the invariant positional criterium that I regard essential in homology. This is based upon neuroanatomical topologically invariant structure and relative position of parts, amplified by mechanistic developmental causal (molecular) analysis [Nieuwenhuys, 2017]. The latter aspect is also crucial, because it attends to how inherited genomes are read out through development (and epigenesis) into brain structure with functional capacities. Briscoe and Ragsdale [2019] prefer mere circuitry and roughly defined functional cell types, without requiring that such isolated characters or features are morphostatic and non-convergent throughout evolution, as is the relevant Bauplan.

In the first place, only well-defined brain parts (rather than "features") can be said to be homologous across species, as long as they are previously defined as constituents at some hierarchical level of the shared Bauplan; note the latter must be given first to allow any comparison to be made (i.e., no Bauplan, and you are comparing pears to oranges, for all you know; however, inside the pear-tree Bauplan you can compare any different sorts of pears). Comparisons are obviously useless and arbitrary if there is no shared Bauplan: why should we compare avian and mammalian brains if they do not have a common Bauplan? One might for the same reason compare the mammalian brain with a fly or spider brain, looking for "func- tionally similar" cell types and circuitry. Any observed individual cellular "feature" - including gene expressions and connections - can be as similar as anybody may subjectively deem them to be, but this still does not tell enough to establish a true homology, because features are not constituent parts of Bauplans. Note that for the same reason practical concentration on isolated features is also potentially problematic in the cladistic approach. This is only saved because the good researcher unconsciously keeps in mind the Bauplan - they only look for variant fingers in what they inexplicably know beforehand are the hands and feet. As was commented above with regard to Owen's [1848] definition of homology, if similarity enters into the notion of homology at all, it is only under the form of a "similar" (that is, equivalent) topologic position within a comparable Bauplan (or a similar or equivalent mechanism of development, which amounts to the same, since fundamental positionally invariant developmental mechanisms provide causal underpinning for the Bauplan structure and any associated variations). True morphologic sameness is thus never based on subjective similarity of isolated characters or features, and even less on similar functions, the province of analogy, but on invariant topology and its causes.

Importantly, whenever the essential comparable topological and mechanistic developmental aspects related to the Bauplan apply, judgements of homology admit absence of any apparent similarity. Take for instance the noses of elephants and whales. Nobody doubts they are homologous as components of the respective very divergent faces, though they cannot be more different anatomically and functionally (including lack of smell sense in whales). However, if we examine the pertinent bones of the respective nasal skeleton, we find that the noses of these two mammalian species are positionally and structurally topologically invariant, that is, are topologically comparable with regard to the Bauplan of cranial bone components, irrespective of substantial anatomic dissimilarity due to quantitative variations suffered in morphogenesis. The fundamental morphologic and ontogenetic invariance is what establishes the homology, not any similarity of apparent features, or how similarly elephants and whales may deal with expulsing water out of their corporal system. We may consider likewise the mouth and the anus of vertebrates. If we dismissed assessing in which part of the Bauplan they are respectively, we might falsely conclude in different species by similarity of isolated characters (sphincter muscles, secretory glands, some excretory functions, etc.) that one item is homologous with the other. This error does not occur normally because even the 
most fervid opponents of the Bauplan concept in comparative anatomy use it subconsciously whenever they look at animal organs (they just wrongly think that, without using a body parts model or Bauplan, they know at first glance what is the mouth and the anus). We sadly have not yet come to such generalized assimilation of the brain Bauplan. We should aim first to agree on the Bauplan before we consider brain connections or hodological functions.

I gather from reading Briscoe et al. [2018] that for them the evolving neocortex is simply "what lies dorsally to the subpallium (i.e., the DT) and serves to be intelligent, using inputs, outputs and intercalated associative cells." They may not realize that their simplistic "dorsal and ventral telencephalon" subdivisions actually represent a very crude bipartite Bauplan based on the most primitive anatomy, while negating more than a century of detailed anatomical studies (Puelles et al. [2017], reproduced in their Fig. 1 the multiple pallial compartments already distinguished by Kalischer [1905], which have turned out to be corroborated by modern molecular analysis).

I thus counter that the topological Bauplan logic needs to be applied consciously and explicitly to all problems of homology in the brain, after full consideration of all available anatomic and developmental data, before any connections, molecular determinants, or cell populations are considered. That is how we identify noses or fingers in nature, and that is how we have to identify neocortex and claustrum homologs (among animal forms sharing a Bauplan). The homologized parts may be found to be similar, partly similar, or far from similar in different secondary features (as happens with noses and fingers), but such differences do not preempt the fundamental homology hypothesis, which also guides any causal mechanistic or functional/hodologic investigations.

As we have seen above (excursus on Karten's theory), the "hodological feature," "molecular feature" or "functional feature" approach to homology that rejects or simplifies rudely any detailed Bauplan considerations (as favored by Karten and followers, as well as by Briscoe and Ragsdale, apparently due to the freedom to speculate thus obtained) remains bare of realistic causal developmental underpinnings, which are even pronounced to be impossible or misleading by some followers [Faunes et al., 2015]. According to the latter authors each brain is a causally unpredictable chaos of molecular and cellular developmental phenomena, which surprisingly does not impede a predictable normal adult form and structure from being achieved in most instances. This supposedly only

Sauropsidian Claustro-Insular Homology can occur thanks to evolutionarily conserved brain connections and related input, output, and associative functions. The hodologist that follows Faunes et al. [2015] vein inexplicably pretends to find order in the assumed developmental and morphologic chaos, apparently because of his/her faith in the unique discriminative power of hodological similarity analysis in adults (see also in this respect the likewise aberrant thesis of Biegler et al. [2021] that comparative functional analysis should also be an inexcusable requirement in embryonic studies). In my discrepant view, genomic structure, development, and morphologic structure are unavoidable preliminary constraints in comparative functional homology thought. The latter may otherwise be diverted into arbitrary directions by any exclusive preference given to human interpretations of hodological results and intuited functions. The functionalists among us cannot let fly their imaginations, leaving aside the causal underpinnings (complex and partly unexplained as these may be), if they want to be scientists.

What about the schema given by Briscoe et al. [2018] on functional neocortical evolution? In the end, do we understand better the mammalian or avian mental capacities by classifying "intelligent DT pallium" into input, output, and associative "cell-homologous" neurons, in the absence of any Bauplan-related positional and developmental consideration? This functional schema is notoriously valid for any brain part in vertebrates and invertebrates, so that all of them turn out to have a Briscoe/ Ragsdale neocortex homolog. I regard this thesis as a noninformative and misleading black box solution that in fact dismisses detailed anatomical, physiological, and pharmacological advances in recent decades and does not examine evolving function in any significant detail (we just start with a neocortex homolog in the earliest vertebrates and perhaps also in invertebrates). The line of thought of editors accepting such conclusions in their journals is a real mystery to me. In what sense is this banal and insufficient idea thought to be a scientific advance in comparative neuroanatomy?

Surprisingly, Briscoe et al. [2018] do admit that the molecular profile they observed at the avian and reptilian mesopallium supports its nature in sauropsids as a "dedicated," i.e., distinct pallial sector. This is exactly what was postulated earlier by me and various collaborators in our original and revised versions of the tetrapartite pallium model [Puelles et al., 2000, 2016a, 2019a; Puelles, 2001, 2014; Medina et al. 2004] but was apparently dismissed a priori by these authors with their subliminally introduced DT concept, whose implicit "neocortical" homogeneity is 
contradicted by this finding. Their own gene mapping mesopallium results should have convinced these authors that their DT is divided at least into three molecularly distinct parts: the mesopallium, the rest of the DVR (itself with subdivisions), and the Wulst (also subdivided). However, Briscoe and Ragsdale do not conclude consequently that this result exposes their initial lumping of mesopallium, nidopallium, and arcopallium into the DVR part of DT and together with the Wulst as an arbitrary measure unsupported by their own molecular data. They did not follow the commonsensical course of thought asking to which particular parts of the mammalian pallium these molecularly "dedicated" parts of sauropsidian DT correspond. Exploring this rationale, they would have met our hypothesis of mesopallium as a selective claustro-insular homolog, perhaps leading them to realize that "neocortex" is not such a dominant or exclusive part of the pallium as they used to think, there existing several other pallium parts to consider comparatively.

They preferred instead to visualize their own "molecularly dedicated" parts of the DT as an irrelevant sort of DT partition allowing them to disregard their topology and morphostatic properties as revealing distinct pallial sectors and insist in their overall sauropsidian DT homology with mammalian neocortex. Molecularly dedicated parts of DT exist, coinciding in fact with our previously formulated pallial sectors, but supposedly remain not significant as such, the "pallial sector" concept being relegated to the uncited past (LPall, VPall; but see Puelles et al. [2017]). Maybe Briscoe et al. [2018] find the pallial sector concept, which is supported by their data, as excessively developmental and distant from their functional interests. Indeed, the significance they report is that they suggest similarities with a class of neocortical neurons showing associative properties known to be manifested in fact by all "intratelencephalic" connectivity. The "similarity" in question is crudely negative, and dissociated from any positional consideration, i.e., the cells are "similar" just in not being either "input- or output-related cells," a situation described as representing "associative" connections. This functional thinking devoid of underlying anatomy passes very lightly over defining any variety of specific functional associative connections in the very complex pallium, lumping them all in this widest definition ("neither input nor output"). As a result they pretend to have demonstrated specifically within the mesopallium "neocortical associative connections," as if associations were not possible within and between other brain parts. Briscoe and Ragsdale thus seem blind to the fact that the functional category of "intratelencephalic associative pallial neurons" does not belong exclusively in mammals to neocortical cells.

Making an exception to their procedure not to distinguish sectors or anatomical parts within the avian or reptilian pallium, no doubt due to the implicit contradiction imposed by the DT concept, Briscoe et al. [2018] undertook a comparative gene expression analysis restricted to the sauropsidian mesopallium. The reason for doing this was not given, since for these authors the mesopallium is just one portion of the DT neocortex homolog; starting from their assumptions, the rationale for studying it separately is not clear. I like to think that it was a reaction to our reports [Puelles et al., 2016a, 2017; Puelles, 2017; Watson and Puelles, 2017] postulating a claustro-insular homolog region in that region of the brain of sauropsids. This hypothesis obviously does not agree with the DT/ neocortex homology hypothesis, and therefore probably was considered to be wrong by the members of the Ragsdale lab. A molecular analysis richer than ours might demonstrate that specific avian and reptilian mesopallium genes are actually more similar as a whole to mammalian neocortex genes than to the restricted mammalian claustro-insular mesocortex domain.

However, Briscoe et al. [2018] surprisingly did not compare their rich mesopallium data one-to-one with the mammalian claustro-insular complex (as they might have done if they wanted to prove wrong our recently published hypothesis standing on scarcely 3 genes: $N r 4 a 2$, $C y p 26 b, C b \ln 2)$. They acknowledge having compared their genes only with the mammalian neocortex as a whole. In my book this territory does not contain the claustrum, since the latter belongs to the claustro-insular mesocortex [Puelles et al., 2019a]. The "functional" hodologic-similarity homology tactic adopted by Briscoe et al. [2018] consisted in comparing exclusively basic connectivity features (input, output, or associative) noted in different parts of the DT with the same features in the mammalian neocortex. No reasons are given for leaving out of the comparison other mammalian pallial parts, including the claustro-insular complex.

Accordingly, not a word of either corroboration or discrepancy with our claustral homology hypothesis was offered in their conclusions. I interpret this very odd aspect as revealing that their Nr4a2 and other data confirmed completely our description of elements in the sauropsidian mesopallium (LPall), which in spatial and molecular pattern are only comparable with the mammalian claustro-insular complex (a part of the mesocortex, not neocortex). This would have refuted their theory on the neocortical nature of the sauropsidian mesopallium, a part of 
the DVR, and thus of their DT. I posit that they secondarily decided to withhold this conclusion. They presented instead their black box theory of mesopallium associative cell populations being "cell-homologous" to neocortex associative cells, which is in fact a good example of a banal analogy, rather than of homology.

In a nutshell, the argument of Briscoe et al. [2018] goes as follows (my brackets and italics):

"Bird intelligence may ... arise from neural circuitry fundamentally similar to that of the neocortex... crossspecies similarities in connectivity led Harvey Karten to propose that input and output $[D T]$ neurons of mammals and birds are homologous [do they mean analogous?] at the cell-type level. Recent studies provided strong support for Karten's cell-type homology hypothesis by demonstrating that input and output $[D T]$ cells express conserved molecular markers [actually functional effector gene markers] - avian input cells are enriched for expression of the neocortical L4 genes KCNH5/EAG2 and RORB, while avian output cells express L5 markers including FEZF2, CACNA1H, and SULF2. Input and output cells, including their defining connections and gene expression profiles, were likely inherited from the last common ancestor of amniotes."

However, we know that no cells or cell features as such are inherited from an ancestor, because each generation has to develop the whole body ex ovo, with variations introduced over millions of years, so that an exact developmental reproduction of the ancestral cellular characteristics cannot be expected. Or are Briscoe and Ragsdale referring to Lamarckian heritage?

The neocortical genes cited by Briscoe et al. [2018] in the passage cited above mostly represent convergently recruited synaptic function-associated molecules that do not identify brain regions but categories of functions, as suggested already by Medina et al. [2013] and Puelles et al. [2017], and this was later corroborated by Tosches et al. [2018].

They admitted this point themselves further below (my brackets and italics):

"We anticipated that transcription factors would be more likely than other classes of molecules to show conservation at the cell-type level [or at the dedicated regional level?] across species. Such factors may also be functionally required for the developmental specification of mesopallium cell-type identity."

This statement contradicts the strange response given by Briscoe and Ragsdale [2019] to the relevant TF-based conclusions of Tosches et al. [2018], copied above literally.

Sauropsidian Claustro-Insular Homology
Let us revise what Briscoe et al. [2018] actually did. After a so-called HOLT analysis (high-to-low ordering of like transcripts) according to DNA sequencing data on selected tissue probes, Briscoe et al. [2018] identified 79 genes expressed and significantly enriched in the avian mesopallium as compared via "filtering" to a nidopallial "input" area and an arcopallial "output" area within the lumped DT domain or preconceived neocortex-homolog avian complex. Given that the nidopallial and arcopallial brain areas they considered are not associated molecularly in terms of TFs to the neocortex, but to the pallial amygdala, as was recently demonstrated by Tosches et al. [2018], then these filters must be regarded as not theoretically significant for filtering out input/output features of mammalian neocortex pyramids. The authors might have filtered the crude mesopallium transcripts with transcripts of Wulst input/output cells since this pallial sector is widely accepted to be comparable to the neocortex. For some reason they did not do that, though it was feasible and obviously preferable. I conclude that the gene filtering procedure was technically defective and possibly preconceived. This only suggests that genes potentially labeling input and output neurons may be mixed in the list jointly with those distinguishing "associative cells."

In their Table 11 listing nearly 80 mesopallium-specific markers, the marker Nr4a2 is characterized in position 54 as "present but not selective" (meaning it is expressed in other parts of the telencephalon apart from the mesopallium, as we already reported; Puelles et al. [2016a]). That is the only reference to our claustrum marker given in the whole paper, leaving the reader in the dark about exactly where this marker was present in their material.

Among the list of 79 mesopallium markers, 14 were TFs. The authors did not explain why they chose to work only with the 5 most abundant TFs present among the first 15 members of the list, plus Emx1 retrieved separately, thus leaving 8 other mesopallial TFs plus 64 other mesopallial genes out of their analysis (their respective expression patterns remained undescribed and unshown). Given that all the 5-6 genes examined label homogeneously the mesopallium (a possible reason why they were selected), it is conceivable that the non-used TFs showed non-homogeneous expression patterns. For instance, $N r 4 a 2$, the gene marker used by us to propose our claustro-insular homology hypothesis for the mesopallium [Puelles et al. 2016a] appears listed among the abandoned TF markers, and it shows non-homogeneous labeling within the mesopallium. Subdivisions are perhaps inconvenient when studying a unitary DT. The authors also 
eliminated Nhlh 2 from the initial selected list because "it did not appear expressed in mouse neocortex" (sic).

In their description of the expression of the final 5 TFs in the mesopallium, Briscoe et al. [2018] employed the recent reclassification of dorsal and ventral mesopallium parts proposed by Jarvis et al. [2013]. This subsumes the classical HD and HI components of the Wulst (old densocellular and intermediate hyperstriatum) under a modified "dorsal mesopallium" concept $(\mathrm{Md})$ introduced by Jarvis et al. [2013], as done likewise by Desfilis et al. [2018]. This in principle lumps the former dorsal and ventral parts of the mesopallium (distinguished for instance in the chick brain atlas of Puelles et al. [2007, 2019b], as well as by Reiner et al. [2004]) within the new concept of "ventral mesopallium" (Mv); we already gave some comments on this confusing terminological reclassification in Puelles et al. [2016a, 2017], as well as above in the present essay. The reorganization just brings under the same "mesopallium" classification the otherwise histologically distinct Wulst and mesopallium areas that happen to co-express the selected 5 TFs, probably among many others. Some of the lumped subdomains of the mesopallium are already known to have selective gene expressions, such as the specific Cbln 2 signal shown selectively by the upper half of the new Mv concept [Reiner et al., 2011], or the selective absence of Nr4a2 signal at the classic HD [Puelles et al., 2016a]. Cyp26b expression is absent at the HI/HD domains [Puelles, 2017; Puelles et al., 2017]. Briscoe et al. [2018] apparently decided that such minutiae did not need to be discussed.

Isolated groups of genes not comprising the whole molecular profile may seem to characterize uniformly an anatomically and developmentally non-unitary brain sector (e.g., as occurs with Hox genes in the hindbrain and spinal cord). To give a joint name to a set of pallial subdomains that happen to share given genes does not convert these subdomains (non-identical in other aspects) into a unitary mass. Imagine that we propose renaming all the hindbrain parts that co-express Hox genes as "spinal cord"; this reclassification may perhaps have some heuristic value, because such genes are indeed variously co-expressed in hindbrain and spinal cord [Tomás-Roca et al., 2016], but the renaming will not eliminate the hindbrain structural peculiarities that we can already recognize, with 10 Hox-expressing rhombomeres (I exclude the isthmo-cerebellar $\mathrm{r} 0$ and $\mathrm{r} 1$ ), and each of them producing different neuronal structures and circuits [Puelles, 2013]. The fact that we continue to distinguish mutual histic/anatomic boundaries between the old and new pallium parts here lumped together on relatively arbitrary molecular grounds by Jarvis et al. [2013] reveals that there must exist also differential (non-shared) gene patterns that create the finer visible architectonic regionalizations of the adult brains. It is not logical to look away from such heterogeneity-creating markers.

One conclusion that the 40 years of molecular era have taught us is that, in most cases in which classic neuroanatomists described an objective histic boundary, it later corresponded to a modern molecular boundary (this is the case of pallial subdivisions and their boundaries; check Kalischer [1905], or his Figure reproduced in $\mathrm{Pu}$ elles et al. [2017]). We also have learned that there exist more molecular borders than limits identified by classic anatomists (as in the obvious case of rhombomeres); of course, a number of classical arbitrarily defined brain limits - such as the classic caudal and rostral limits of the midbrain - have been refuted by accruing molecular results [Puelles, 2019]. Time will accordingly tell us which mesopallium molecular definition is stronger and more useful. I momentarily remain with the classic one, though acknowledging readily that the avian $\mathrm{HI} / \mathrm{HD}$ (parts of DPall in my analysis) share a number of genes with the classic mesopallium (LPall); this is surely not true for all genes these compartments express, since we already know some exceptions, like Nr4a2 and Cyp26b.

Briscoe et al. [2018] say that they only checked in the mouse neocortex whether the signal of the chosen 5 avian mesopallial TFs was present there. Their conclusions neatly confirmed the preconceived expectations of the main authors: mesopallial neurons share the selected 5 markers with some neocortical associative neurons (among a total of 14 TFs in their Table S1); neither this minority proportion nor the reasons for exclusion of the other 8 TFs from the analysis were discussed (but see below).

Since the authors did not use their opportunity to compare the studied markers with the mouse claustrum and insula (or, if they did, as I suspect, they decided to say nothing after looking at the data), I checked all the 14 mesopallium-enriched TFs in the Allen Developing Mouse Brain Atlas at embryonic and postnatal stages, looking for expression within either DPall (neocortex) and/or LPall (claustro-insular complex). There were 3 mesopallial TFs (Nhlh2, Pkib, and Tfap2d) that lacked both cortical and claustro-insular expression in the mouse. This means that the avian mesopallium, even with the addition of dorsopallial HI and HD portions, is different from both neocortex and the mammalian claustroinsular complex in some molecular aspects. As regards the other $11 \mathrm{TFs}$, I found the following results: 
Out of the 5 used TFs, all of them (Id2, Satb2, Foxp1, $B c l 11 a$, and Emx1) showed expression in the mouse LPall (i.e., the claustro-insular complex). The signal appeared specifically either in the claustrum or the insula, or in both, and sometimes expression was also present in migrated claustral derivatives such as subplate cells, Arimatsu cells in parietal layers 5/6, or the EPd nucleus [Puelles, 2014; Watson and Puelles, 2017].

Out of the 6 remaining abandoned TFs (including $\mathrm{Nr} 4 \mathrm{a} 2$ and $\mathrm{Nr} 4 \mathrm{a} 3$ ), all of them likewise showed expression in the LPall (claustrum, insula or both, as well as in Arimatsu cells migrated to the parietal layers $5 / 6$, the corresponding ventricular zone, or, in 1 case, the marker was positive only in the cortical subplate and the EPd nucleus (similarly to Cgtf; Watson and Puelles [2017]); these various elements represent tangentially migrated claustral cells according to my analysis in Puelles [2014].

The 11 mesopallial TF markers additionally showed various expression subdomains in some DPall, VPall (piriform cortex), MPall (hippocampus, entorhinal cortex), or amygdalar subregions. This just indicates that the regulation of their expression is complex and pleiotropic. Most cases of labeled DPall (neocortical) domains (7 TFs $=63.63 \%$ ) were represented by both deep and upper layer neurons, whereas selective deep layer labeling was found only in 2 TF cases $(=18.18 \%)$; in another case the labeled deep-layer cells were interpreted specifically as tangentially migrated Arimatsu claustral elements $[\mathrm{Pu}-$ elles, 2014] due to their restriction to layers $5 / 6$ of the parietal cortex (Fig. 2) [Arimatsu et al., 1994].

This claustro-insular checking in the mouse that Briscoe et al. [2018] were unable or unwilling to do shows that all 11 TFs enriched in the avian mesopallium (100\%) that were also present in the neocortex were likewise expressed differentially in the mouse claustro-insular complex and/ or some claustral migrated derivatives [Puelles, 2014; Watson and Puelles, 2017], with or without additional pallial expression sites. These TFs would need to be filtered to eliminate any genes possibly corresponding selectively to NI or ND (possible parts of DPall, after all, irrespective of semantic tricks). In $2 \mathrm{TF}$ cases ( $\mathrm{Nr} 4 a 3, \mathrm{Bhl}$ he40) the labeling was clearly restricted to the insula; in 2 other cases (Nr4a2, Fosl2) the signal appeared selectively in the claustrum, the dorsal endopiriform nucleus, deep parietal Arimatsu cells, and the subplate (EPd, Sp); all these extra populations migrate out of the claustral primordium [Puelles, 2014; Watson and Puelles, 2017]. In 1 case (Tle4) the claustrum proper was unlabeled, but layer $6 \mathrm{~b}$ (the subplate) of the insula and the entire subplate plus migrated EPd were labeled. In the case of Atoh8 the ven-

Sauropsidian Claustro-Insular Homology tricular zone of both LPall and DPall was found to be positive at early stages.

It is really remarkable that Briscoe et al. [2018] saw fit not to say anything about these aspects which they must have observed, and particularly said nothing about the mesopallial claustrum marker we had used, Nr4a2, although they had it in their list of mesopallium markers (their Table S1). It just appears marked succinctly as "expressed, but not mesopallium-specific," which implies someone looked at its in situ mappings. The fact that expression also appears in the hippocampus and the arcopallium (as shown in Puelles et al. [2016a]) is not a valid reason to withhold comparing the avian mesopallial data with the mouse claustro-insular complex. Moreover, Briscoe et al. [2018] also did not show their mappings of the alligator mesopallial genes.

Recognition of a separate claustro-insular-like mesopallium labeling pattern would corroborate our claustrum homology hypothesis, and automatically would center the comparative attention upon the claustro-insular aspects rather than on the notion of "associative neocortex cells." The mammalian claustro-insular complex appears labeled by all 11 avian mesopallial TFs expressed in mouse present in the gene list of these authors. The markers not described or illustrated by Briscoe et al. [2018] label differentially the mouse insula, the claustrum, and its migrated derivatives. For authors that are nominally interested in distinct cell types it was a peculiar decision to eliminate this evidence.

\section{E.D. Jarvis and Colleagues}

I would have ended about here this commentary on recent publications touching directly or indirectly our claustro-insular homology hypothesis for sauropsids and mammals, though I have read a few other reports that might have been considered as well (e.g., Gedman et al. [2021], from the Jarvis lab). However, limits of space recommend we leave this critique for another occasion. I nevertheless feel obliged to add a brief reference to the recent Biegler et al. [2021] report also coming from the Jarvis lab. Here the authors pretend to refute our claustroinsular hypothesis irrespective of both the Tosches et al. [2018] data and our developmental chicken data [Puelles 2014, 2017; Puelles et al., 2016a; Watson and Puelles, 2017] by looking mainly at functional labeling of adult songbird brains. They suggestthat one cannot deduce homology without performing functional studies, even in the case of embryonic material. One guesses that such "embryonic functional studies" need to be done without paying much attention to anatomical subdivisions. They also 
affirm that the claustrum marker Nr4a2 is not a TF involved in early tissular specification because they can induce its expression functionally in adults. However, it cannot be negated that the gene is spontaneously expressed in the mouse and chicken claustral populations as soon as the neurons become postmitotic, at which moment they surely are not functional (see Fig. 3) [Puelles, 2014; Puelles et al., 2016a). The hardly classic emphasis on function by these authors, held to be a necessary methodological approach for comparative brain analysis, outdoes the comparable pretensions of Faunes et al. [2015], mentioned above (let us remember once more Owen's homology definition cited above).

I acknowledge that Biegler et al. [2021] included a fair summary of our findings and also reported having examined the adult $\mathrm{Nr} 4 a 2$ expression pattern, although they insisted on using the highly controversial alternative Jarvis Bauplan for the avian pallium which we already criticized in detail [Jarvis et al., 2013; Puelles et al., 2017]; curiously, they do not argue against any of those critical comments, so I will not repeat them here. Biegler et al. [2021] limit their critical comments on avian mesopallial $\mathrm{Nr} 4 \mathrm{a} 2$ expression to saying that they do not see in adults such a widespread mesopallial expression of $\mathrm{Nr} 4 a 2$ as we described in embryos. This maturational feature was already disclosed by us [Puelles et al., 2016a] and it just tells us that this gene is not particularly active in the adult. They thus corroborate in essence our avian mesopallium description, establishing accordingly a significantly higher scientific standard than Briscoe et al. [2018]. The only problem they see is that our data were embryonic and not functional, whereas theirs were functional on adults. I would love it if all my problems were like this one.

They do make a strong point of not believing the evidence we showed [Puelles, 2014, 2017; Puelles et al., 2016a, 2017; Watson and Puelles, 2017] that some avian claustral populations migrate tangentially into DPall and VPall, similarly as mammalian counterparts do in the mouse (Fig. 7a, b). They adduce the Bruguier et al. [2020] publication (where I appear as a senior coauthor) in which it is affirmed that no migration whatsoever occurs between the avian LPall and the DPall. This indeed throws a shadow on anything we said before on such migrations. The negative evidence on the postulated migration that was emphasized by Bruguier et al. [2020] without my acquiescence originated in the earlier publication of GarcíaMoreno et al. [2018]. My argument that this had important errors of interpretation (which I detailed) and was demonstrably contradicted by available data (see below) was not accepted, without leaving me time to withdraw from authorship. Indeed, chick embryo experiments performed by F. Garcia-Moreno himself with his own construct and methodology [García-Moreno et al., 2014] during a research stay he did in my laboratory had produced cases in which a migration from LPall into DPall and VPall was evident. I requested to Molnar without success that this image be included in the publication, with the explanation that some experiments gave positive results and some gave negative ones. In our cases I had guided F. García-Moreno personally to the appropriate locus of LPall labeling (not easy to locate in 4-day-old embryos). Apparently, the labeling sites were not the same in the earlier negative experiments he had done at home. Such positive experiments (already shown photographically in Puelles [2017] and Watson and Puelles [2017], and reproduced here once more in Fig. 7a) wholly confirmed our published descriptive analysis (see Fig. 7b). This is why I appear as coauthor, but do not support, nor like to cite at all, the Bruguier et al. [2020] publication.

On the whole, I am satisfied that the claustrum homology hypothesis has received significant outside support, and it has helped the landmark recognition of the reptilian claustral deep-sleep inducing nature in parallel to the mouse equivalent claustral function (thus even observing functional homology, if that exists without a functional Bauplan). This satisfying feedback was mixed sometimes with partial divergences on the semantics or component details of the pallium model, but this is something that time will cure, and we are already looking at the next stage represented by the cortical concentric-ring model $[\mathrm{Pu}-$ elles et al., 2019a] and our recent work on the amygdala. There was also the group of a priori antagonistic Karten believers around Ragsdale whose analysis could have been feared, if we were wrong. However, they voluntarily surrendered the controversy by their meaningful silence on any contradictory data, and their preposterous definition of homology based on the "sum of both similar and dissimilar features" in response to the data from Tosches et al. [2018], strongly contradictory to their thesis. They clearly did not find any mentionable objection to our mesopallium comparative analysis, rather the opposite, as I argued, but preferred to present once more their preconceived holistic dogma equating non-mammalian pallium simply with mammalian neocortex. This trend brings brain homology analysis to an all-time scientific minimum, in my opinion. Briscoe et al. [2018] in essence propose a pallial black box model with many positionally illidentified elements inside, defined simply as "input, output, and associative neocortical neurons" (because it is postulated ab initio that they all are neocortical). Their 
postulated value is presented as unrelated to the fact that they may differentially reside developmentally and phylogenetically within molecularly "dedicated" dorsopallial, lateropallial, ventropallial, or amygdalopallial pallium domains, not accepted ab initio by these authors as constituting a useful comparative Bauplan. Their report was commented on laudatorily by Maler [2018].

If intelligence just depends on having assorted "associative neurons" in the pallium then all sorts of animals down to cyclostomes must be equally "intelligent" as birds and mammals (see other reports in the same volume). One can jump to the manifestly absurd conclusion that vertebrates just have in their pallium a very complex and chaotic neocortex with gene patterns and parts that do not need to be shown, in place of a differentially evolved finely subdivided pallium achieving variations around the conserved Bauplan theme. We do not need functional generalities but specificities, and are therefore interested in well-systematized cellular and molecular structure rather than in their negation.

Consistency and coherence of well-selected data also needs an associated sound theoretic superstructure [Bunge, 1973, 2006; Mahner and Bunge, 1997; Nieuwenhuys, 2017). The next generations of neuroscientists occupying positions in university departments, laboratories, journals, and funding agencies will be involved in that endeavor.

\section{Statement of Ethics}

All original experimental protocols and handling, use, and care of laboratory animals were conducted in compliance with the current normative standards of the European Union (Directive 2010/63/EU), the Spanish Government (Royal Decree 1201/2005 and 53/2013; Law 32/107), and with the approval of the University of Murcia committee for animal experimental ethics (No. A13170406).

\section{Conflict of Interest Statement}

The author has no conflicts of interest to declare.

\section{Funding Sources}

The support of the Spanish Ministry of Economy and Competitiveness grant BFU2014-57516P (with European Community FEDER support), and a Seneca Foundation (Autonomous Community of Murcia) Excellency Research contract, reference: 19904/ GERM/15; project name: Genoarchitectonic Brain Development and Applications to Neurodegenerative Diseases and Cancer (to L.P.), by Seneca Foundation (5672 Fundación Séneca) are acknowledged. University of Murcia, VAT: ESQ3018001B.

\section{References}

Abellán A, Desfilis E, Medina L. Combinatorial expression of Lef1, Lhx2, Lhx5, Lhx9, Lmo3, Lmo4, and Proxi helps to identify comparable subdivisions in the developing hippocampal formation of mouse and chicken. Front Neuroanat. $2014 \mathrm{Jul} \mathrm{4;8:59.}$

Arai Y, Pierani A. Development and evolution of cortical fields. Neurosci Res. 2014;86:66-76.

Arimatsu Y, Nihonmatsu I, Hirata K, TakiguchiHayashi K. Cogeneration of neurons with a unique molecular phenotype in layers $\mathrm{V}$ and VI of widespread lateral neocortical areas in the rat. J Neurosci. 1994;14:2020-31.

Atoji Y, Wild JM. Afferent and efferent projections of the mesopallium in the pigeon $(\mathrm{Co}-$ lumba livia). J Comp Neurol. 2012;520:71741.

Atoji Y, Sarkar S, Wild JM. Differential projections of the densocellular and intermediate parts of the hyperpallium in the pigeon ( $\mathrm{Co}$ lumba livia). J Comp Neurol. 2018;526:14665.

Bayer SA, Altman J. Development of the endopiriform nucleus and the claustrum in the rat brain. Neuroscience. 1991;45:391-412.

Biegler MT, Cantin LJ, Scarano DL, Jarvis ED. Controlling for activity-dependent genes and behavioral states is critical for determining brain relationships within and across species. J Comp Neurol. 2021;529(12):3206.

Borello U, Pierani A. Patterning the cerebral cortex: traveling with morphogens. Curr Opin Genet Dev. 2010;20:408-15.

Briscoe SD, Albertin CB, Rowell JJ, Ragsdale CW. Neocortical association cell types in the forebrain of birds and alligators. Curr Biol. 2018; 28:686-96.e6.

Briscoe SD, Ragsdale CW. Molecular anatomy of the alligator dorsal telencephalon. J Comp Neurol. 2018;526:1613-46.

Briscoe SD, Ragsdale CW. Evolution of the chordate telencephalon. Curr Biol. 2019;29:R64762.

Bruce LL. Evolution of the nervous system in reptiles. In: Kaas JH, Bullock T, editors. Evolution of nervous systems: a comprehensive reference. San Diego: Nonmammalian Vertebrates Academic Press; 2007. Vol. 2; p. 125-56.

Bruce LL. The puzzle of forebrain evolution. Brain Behav Evol. 2012;79:141-3.

Bruce LL, Neary TJ. Afferent projections to the ventromedial hypothalamic nucleus in a lizard, Gekko gecko. Brain Behav Evol. 1995;46: 14-29.
Bruguier H, Suarez R, Manger P, Hoerder-Suabedissen A, Shelton AM, Oliver DK, et al. In search of common developmental and evolutionary origin of the claustrum and subplate. J Comp Neurol. 2020;528(17):2956.

Bunge M. Method, model and matter. Dordrecht: D.Reidel; 1973.

Bunge M. Chasing reality. Strife over realism. Toronto: University of Toronto Press; 2006.

Butler AB, Reiner A, Karten HJ. Evolution of the amniote pallium and the origins of mammalian neocortex. Ann N Y Acad Sci. 2011;1225: 14-27.

Chen CC, Winckler CM, Pfenning AR, Jarvis ED, et al. Molecular profiling of the developing avian telencephalon: regional timing and brain subdivision continuities. J Comp Neurol. 2013;521:3666-701.

Dart RA. The dual structure of the neopallium: its history and significance. J Anat. 1934;69:319.

Desfilis E, Abellán A, Sentandreu V, Medina L. Expression of regulatory genes in the embryonic brain of a lizard and implications for understanding pallial organization and evolution. J Comp Neurol. 2018;526:166202 
Dugas-Ford J, Ragsdale CW. Levels of homology and the problem of neocortex. Annu Rev Neurosci. 2015;38:351-68.

Fang C, Wang H, Naumann RK. Developmental patterning and neurogenetic gradients of claustrum nuclei and claustrum-like cell types in the rat cerebral cortex. Front Neuroanat. 2021;15:786329.

Faunes M, Botelho JF, Ahumada Galleguillos P, Mpodozis J. On the hodological criterion for homology. Front Neurosci. 2015;9:223.

Garcia-Calero E, Martínez-de-la-Torre M, Puelles L. A radial histogenetic model of the mouse pallial amygdala. Brain Struct Funct. 2020;225:1921-56.

Garcia-Calero E, López-González L, Martínezde-la-Torre M, Fan C-M, Luis Puelles L. Sim1-expressing cells illuminate the origin and course of migration of the nucleus of the lateral olfactory tract in the mouse amygdala. Brain Res Funct. 2021;226(2):519.

Garcia-Calero E, Puelles L. Lhx9 gene expression during development of the mouse anterior amygdalar radial unit. Brain Res Funct. 2021; 226(2):575.

García-Moreno F, Vasistha NA, Begbie J, Molnár Z. CLoNe is a new method to target single progenitors and study their progeny in mouse and chick. Development. 2014;141:15891598.

García-Moreno F, Anderton E, Jankowska M, Begbie J, Encinas JM, Irimia M, et al. Absence of tangentially migrating glutamatergic neurons in the developing avian brain. Cell Rep. 2018; 22:96-109.

Gedman G, Haase B, Durieux G, Biegler M, Fedrigo $\mathrm{O}$, Jarvis $\mathrm{ED}$. As above, so below: whole transcriptome profiling supports the continuum hypothesis of avian dorsal and ventral pallium organization. J Comp Neurol. 2021 Aug;529(12):322; this version posted.

Goffinet AM, Bar I, Bernier B, Trujillo C, Raynaud A, Meyer G. Reelin expression during embryonic brain development in lacertilian lizards. J Comp Neurol. 1999;414:533-50

Goffinet AM, Daumerie C, Langerwerf B, Pieau C. Neurogenesis in reptilian cortical structures. H3-thymidine autoradiographic analysis. J Comp Neurol. 1986;243:106-116.

Holmgren N. Points of view concerning forebrain morphology in higher vertebrates. Acta Zool Stockh. 1925;6(3):413-59.

Jacobshagen E. Allgemeine vergleichende formenlehre der tiere. Leipzig: Werner Klinkhardt; 1925.

Jacobshagen E. Zur Reform der Allgemeinen Vergleichenden Formenlehre der Tiere. Jena: Gustav Fischer; 1927

Jarvis ED, Yu J, Rivas MV, Horita H, Feenders G, Whitney $\mathrm{O}$, et al. Global view of the functional molecular organization of the avian cerebrum: mirror images and functional columns. J Comp Neurol. 2013;521:3614-65.

Kalischer O. Das Grosshirn der Papageien in anatomischer und physiologischer Beziehung. Berlin: Verlag der Königlichen Akademie der Wissenschaften; 1905.
Källén B. On the ontogeny of the reptilian forebrain. Nuclear structures and ventricular sulci. J Comp Neurol. 1951;95:307-348.

Källén B. On the nuclear differentiation during ontogenesis in the avian forebrain and some notes on the amniote strioamygdaloid complex. Acta Anat. 1953;17:72-84.

Källén B. Notes on the mode of formation of brain nuclei during ontogenesis. J Comp Neurol. 1955:747-756.

Källén B. Embryogenesis of brain nuclei in the chick telencephalon. Result Anat Development. 1962;36:62-82.

Karten HJ. The organization of the avian telencephalon and some speculations on the phylogeny of the amniote telencephalon. Ann N Y Acad Sci. 1969;167(1):164-79.

Karten HJ. Vertebrate brains and evolutionary connectomics: on the origins of the mammalian 'neocortex'. Philos Trans R Soc Lond B Biol Sci. 2015;370:20150060.

Kuhlenbeck H. Über die Homologien der Zellmassen im Hemisphärenhirn der Wirbeltiere. Folia Anatom Japon. 1924;2(5):325-64.

Kuhlenbeck H. Vorlesungen über das Zentralnervensystem der Wirbeltiere. Jena: Gustav Fischer; 1927

Kuhlenbeck H. Propedeutics to comparative neurology. In: The central nervous system of vertebrates. Basel: S. Karger; 1967.

Kuhlenbeck H. Part II: overall morphological pattern. In. The central nervous system of vertebrates. Basel: S. Karger; 1973.

Mahner M, Bunge M. Foundations of biophilosophy. Berlin: Springer; 1997.

Maler L. Brain evolution: intelligence without a cortex. Curr Biol. 2018;28(5):R213-15.

Medina L. Field homologies. In: Kaas JH, Striedter GF, Rubenstein JLR, editors. Evolution of nervous systems: a comprenhensive reference. Amsterdam: Academic Press-Elsevier; 2007. Vol. 1; p. 73-87.

Medina L, Abellán A, Desfilis E. A never-ending search for the evolutionary origin of the neocortex: rethinking the homology concept. Brain Behav Evol. 2013;81:150-3.

Medina L, Abellán A, Desfilis E. Evolution of pallial areas and networks involved in sociality: comparison between mammals and sauropsids. Front Physiol. 2019;10:894.

Medina L, Legaz I, González G, de Castro F, Rubenstein JL, Puelles L. Expression of Dbx1, neurogenin 2 , semaphorin $5 \mathrm{~A}$, cadherin 8 , and Emx1 distinguish ventral and lateral pallial histogenetic divisions in the developing mouse claustroamygdaloid complex. J Comp Neurol. 2004;474:504-23.

Narikiyo K, Mizuguchi R, Ajima A, Shiozaki M, Hamanaka H, Johansen JP, et al. The claustrum coordinates cortical slow-wave activity. Nat Neurosci. 2020;23:741-53.

Naumann RK, Ondracek JM, Reiter S, Shein-Idelson M, Tosches MA, Yamawaki TM, et al. The reptilian brain. Curr Biol. 2015;25:R317-21.
Naumann RK, Laurent G. Function and evolution of the reptilian cerebral cortex. In: Kaas JH, Striedter G, editors. Evolution of nervous systems. 2nd ed. Oxford: Academic Press; 2017. Vol. 1; p. 491-518.

Nieuwenhuys R. Principles of current vertebrate neuromorphology. Brain Behav Evol. 2017; 90(2):117-30.

Nieuwenhuys R, Puelles L. Towards a new neuromorphology. Berlin: Springer; 2016.

Norimoto H, Fenk LA, Li HH, Tosches MA, Gallego-Flores T, Hain D, et al. A claustrum in reptiles and its role in slow-wave sleep. $\mathrm{Na}$ ture. 2020;578:413-8.

Owen R. On the archetype and homologies of the vertebrate skeleton. London: Richard and John E. Taylor; 1848.

Pattabiraman K, Golonzhka O, Lindtner S, Nord AS, Taher L, Hoch R, et al. Transcriptional regulation of enhancers active in protodomains of the developing cerebral cortex. Neuron. 2014;82:989-1003.

Puelles L. Thoughts on the development, structure and evolution of the mammalian and avian telencephalic pallium. Philos Trans R Soc Lond B Biol Sci. 2001;356:1583-98.

Puelles L. Pallio-pallial tangential migrations and growth signaling: new scenario for cortical evolution? Brain Behav Evol. 2011;78:108-27.

Puelles L. Plan of the developing vertebrate nervous system relating embryology to the adult nervous system (prosomere model, overview of brain organization). In: Rubenstein JLR, Rakic P, editors. Comprehensive developmental neuroscience: patterning and cell type specification in the developing CNS and PNS. Amsterdam: Academic Press; 2013. p. 187209.

Puelles L. Development and evolution of the claustrum. In: Smythies JR, Edelstein LR, Ramachandran VS, editors. The claustrum: structural, functional and clinical neuroscience. San Diego: Elsevier Academic Press; 2014. p. 119-76.

Puelles L. Comments on the updated tetrapartite pallium model in the mouse and chick, featuring a homologous claustro-insular complex. Brain Behav Evol. 2017;90(2):171-89.

Puelles L. Survey of midbrain, diencephalon, and hypothalamus neuroanatomic terms whose prosomeric definition conflicts with columnar tradition. Front Neuroanat. 2019;13:20.

Puelles L, Alonso A, García-Calero E, Martínezde-la-Torre M. Concentric ring topology of mammalian cortical sectors and relevance for patterning studies. J Comp Neurol. 2019a; 527:1731-52.

Puelles L, Ayad A, Alonso A, Sandoval JE, Martínez-de-la-Torre M, Medina L, et al. Selective early expression of the orphan nuclear receptor Nr4a2 identifies the claustrum homolog in the avian mesopallium: impact on sauropsidian/mammalian pallium comparisons. J Comp Neurol. 2016a;524:665-703. 
Puelles L, Harrison M, Paxinos G, Watson C. A developmental ontology for the mammalian brain based on the prosomeric model. Trends Neurosci. 2013;36:570-8.

Puelles L, Kuwana E, Puelles E, Rubenstein JL. Comparison of the mammalian and avian telencephalon from the perspective of gene expression data. Eur J Morphol. 1999;37:13950.

Puelles L, Kuwana E, Puelles E, Bulfone A, Shimamura K, Keleher J, et al. Pallial and subpallial derivatives in the embryonic chick and mouse telencephalon, traced by the expression of the genes Dlx-2, Emx-1, Nkx-2.1, Pax6, and Tbr-1. J Comp Neurol. 2000;424:40938.

Puelles L, Martinez-de-la-Torre M, Martinez S, Watson C, Paxinos G. The chick brain in stereotaxic coordinates and alternate stains. 1st ed. New York: Academic Press; 2007.

Puelles L, Martinez-de-la-Torre M, Martinez S, Watson C, Paxinos G. The Chick brain in stereotaxic coordinates and alternate stains. 2nd ed. New York: Academic Press; 2019b.

Puelles L, Medina L. Field homology as a way to reconcile genetic and developmental variability with adult homology. Brain Res Bull. 2002; 57:243-55.

Puelles L, Medina L, Borello U, Legaz I, Teissier A, Pierani A, et al. Radial derivatives of the mouse ventral pallium traced with $D b x 1-L a c Z$ reporters. J Chem Neuroanat. 2016b;75:2-19.

Puelles L, Sandoval JE, Ayad A, del Corral R, Alonso A, Ferran JL, et al. The pallium in reptiles and birds in the light of the updated tetrapartite pallium model. In: Kaas J, editor. Evolution of nervous systems. 2nd ed. Oxford: Elsevier; 2017. Vol. 1. p. 519-55.

Ramos JMJ. Disconnection of the perirhinal and insular cortices severely disrupts taste neophobia. Neurobiol Learn Mem. 2020;175: 107324.

Reiner A, Perkel DJ, Bruce LL, Butler AB, Csillag A, Kuenzel W, et al. The avian brain nomenclature forum: terminology for a new century in comparative neuroanatomyrevised nomenclature for avian telencephalon and some related brainstem nuclei. J Comp Neurol. 2004;473:E1-414.

Reiner A, Yamamoto K, Karten HJ. Organization and evolution of the avian forebrain. Anat Rec A Discov Mol Cell Evol Biol. 2005;287:1080102.
Reiner A, Yang M, Cagle MC, Honig MG. Localization of cerebellin-2 in late embryonic chicken brain: implications for a role in synapse formation and for brain evolution. J Comp Neurol. 2011;519:2225-51.

Remane A. Die Grundlagen des natürlichen Systems der vergleichenden Anatomie und der Phylogenetik. 1. Theoretische Morphologie und Systematik. Leipzig: Geest und Portig; 1952.

Sanides F. Comparative architectonics of the neocortex of mammals and their evolutionary interpretation. In: Petras JM, Noback CR, editors. Comparative and evolutionary aspects of the vertebrate central nervous system. New York: The New York Academy of Sciences; 1969. Vol. 167. p. 404-23.

Shein-Idelson M, Ondracek JM, Liaw HP, Reiter S, Laurent G. Slow waves, sharp waves, ripples, and REM in sleeping dragons. Science. 2016;352:590-5.

Smith-Fernandez A, Pieau C, Repèrant J, Boncinelli E, Wassef M. Expression of the Emx-1 and $D l x-1$ homeobox genes define three molecularly distinct domains in the telencephalon of mouse, chick, turtle, and frog embryos: implications for the evolution of telencephalic subdivisions in amniotes. Development. 1998;125:2099-111.

Smythies JR, Edelstein LR, Ramachandran VS. The claustrum. Structural, functional and clinical neuroscience. San Diego: Academic Press; 2014.

Striedter GF. The telencephalon of tetrapods in evolution. Brain Behav Evol. 1997;49:179213.

Striedter GF, Keefer BP. Cell migration and aggregation in the developing telencephalon: pulse-labeling chick embryos with bromodeoxyuridine. J Neurosci. 2000;20:8021-30.

Striedter GF, Northcutt RG. Brains through time: a natural history of vertebrates. New York: Oxford University Press; 2020.

Suzuki IK, Kawasaki T, Gojobori T, Hirata T. The temporal sequence of the mammalian neocortical neurogenetic program drives mediolateral pattern in the chick pallium. Dev Cell. 2012;22:863-70.
Suzuki IK, Hirata T. Neocortical neurogenesis is not really "neo": a new evolutionary model derived from a comparative study of chick pallial development. Dev Growth Differ. 2013;55:173-87.

Suzuki IK, Hirata T. A common developmental plan for neocortical gene-expressing neurons in the pallium of the domestic chicken Gallus gallus domesticus and the Chinese softshell turtle Pelodiscus sinensis. Front Neuroanat. 2014;8:20.

Tomás-Roca L, Corral-San-Miguel R, Aroca P, Puelles L, Marín F. Crypto-rhombomeres of the mouse medulla oblongata, defined by molecular and morphological features. Brain Struct Funct. 2016;221:815-38.

Tosches MA, Yamawaki TM, Naumann RK, Jacobi AA, Tushev G, Laurent G. Evolution of pallium, hippocampus, and cortical cell types revealed by single-cell transcriptomics in reptiles. Science. 2018;360:881-8.

Tsai HM, Garber BB, Larramendi LM. 3H-thymidine autoradiographic analysis of telencephalic histogenesis in the chick embryo. I. Neuronal birthdays of telencephalic compartments in situ. J Comp Neurol. 1981;198:275292.

Wang WZ, Oeschger FM, Montiel JF, GarcíaMoreno F, Hoerder-Suabedissen A, Krubitzer $\mathrm{L}$, et al. Comparative aspects of subplate zone studied with gene expression in sauropsids and mammals. Cereb Cortex. 2011;21:2187203.

Watson C, Puelles L. Developmental gene expression in the mouse clarifies the organization of the claustrum and related endopiriform nuclei. J Comp Neurol. 2017;525:1499-508.

Wullimann MF. Should we redefine the classic lateral pallium? J Comp Neurol. 2017a;525: 1509-13.

Wullimann MF. Names matter: commentary on Luis Puelles' article. Brain Behav Evol. 2017b; 90:190.

Yoshihara Y. Functional analysis of an unexplored brain region: the claustrum. Brain Nerve. 2021;73:267-272.

Zilles K, Wree A. Cortex: areal and laminar structure. In: Paxinos G, editor. The rat nervous system. Forebrain and midbrain. North Ryde: Academic Press Australia; 1985. Vol. 1; p. pp375-415. 